\title{
Spectral functions of strange vector mesons in asymmetric hyperonic matter
}

\author{
Amruta Mishra* \\ Department of Physics, Indian Institute of Technology, \\ Delhi, Hauz Khas, New Delhi - 110 016, India \\ S.P. Misra ${ }^{\dagger}$ \\ Institute of Physics, Bhubaneswar - 751005, India
}

\begin{abstract}
We study the medium modifications of the spectral functions as well as production cross-sections of the strange vector mesons $\left(\phi, K^{*}\right.$ and $\left.\bar{K}^{*}\right)$ in isospin asymmetric strange hadronic matter. These are obtained from the in-medium masses of the open strange mesons and the decay widths $\phi \rightarrow K \bar{K}$, $K^{*} \rightarrow K \pi$ and $\bar{K}^{*} \rightarrow \bar{K} \pi$ in the hadronic medium. The decay widths are computed using a field theoretic model of composite hadrons with quark/antiquark constituents, from the matrix element of the light quark-antiquark pair creation term of the free Dirac Hamiltonian between the initial and final states. The matrix element is multiplied with a coupling strength parameter for the light quark-antiquark pair creation, which is fitted to the observed vacuum decay width of the decay process. There are observed to be substantial modifications of the spectral functions as well as production cross-sections of these vector mesons due to isospin asymmetry as well as strangeness of the hadronic medum at high densities. These studies should have observable consequences, e.g. in the yield of the hidden and open strange mesons arising from the isospin asymmetric high energy heavy ion collisions at the Compressed baryonic matter (CBM) experiments at the future facility at GSI.
\end{abstract}

*Electronic address: amruta@physics.iitd.ac.in

${ }^{\dagger}$ Electronic address: misrasibaprasad@gmail.com 


\section{INTRODUCTION}

The study of the in-medium properties of the strange mesons is relevant for the heavy ion collision experiments, as these can affect the experimental observables, e.g., the yield, spectra and collective flow of these mesons in the strongly interacting matter created from high energy nuclear collisions [1]. The understanding of the interactions of the strange mesons with nuclei and nuclear matter is needed for the study of kaonic atoms [2], the experimental observables of the heavy ion collision experiments, as well as the composition of matter in the interior of the neutron stars [3]. The possibility of antikaon condensation [4] in the interior of the neutron stars, due to the mass drop of the antikaons arising from attractive interaction with the nucleons, initiated a lot of work on the study of in-medium properties of these open strange mesons. It is also important to study the effects of the isospin asymmetry of the hadronic medium on the hadron properties in the context of heavy ion collision experiments as the initial system, consisting of heavy ions has large asymmetry in the number of protons and neutrons. The isospin asymmetry effects can affect observables like the $\pi^{-} / \pi^{+}$ratio, the $n / p$ ratio, the $\Delta^{-} / \Delta^{++}$ratio for neutron rich heavy ion collisions. The effects of the medium modifications of the kaons and antikaons in the isospin asymmetric hadronic matter can show in the experimental observables, e.g., the $K^{+} / K^{0}$, the $K^{-} / \bar{K}^{0}$, the $K^{*+} / K^{* 0}$ and the $K^{*-} / \overline{K^{* 0}}$ ratios, as well as the yield, spectra and collective flow of the strange mesons.

The kaons and antikaons in the hadronic medium have been studied extensively in the literature. In Ref. [4], the interactions of the kaons and antikaons with the nucleons arise due to the leading order vectorial Weinberg-Tomozawa term and the attractive kaon-nucleon sigma term at the sub-leading order within the chiral perturbation theory. The $K$ and $\bar{K}$ mesons have also been studied using a meson exchange model [5, 6] incorporating the interactions of these open strange mesons to the meson fields $(\sigma, \omega, \rho)$ similar to the interactions of the baryons to these meson fields in Quantum Hadrodynamics (QHD) model [7]. Within the framework of the Quark meson coupling (QMC) model, the light $(q=(u, d))$ quark (antiquark) constituents of a hadron, $h$, confined inside a bag of radius $R_{h}\left(R_{h}^{*}\right)$ in the vacuum (hadronic medium), interact via the exchange of the light $(\sigma, \omega, \rho)$ mesons. The scalar potential, $V_{s}^{h}$, experienced by the hadron in the medium, which is equal to the mass shift $\left(m_{h}^{*}-m_{h}\right)$ of the hadron, has dominant contributions from the scalar potentials $\left(V_{\sigma}^{q}=g_{\sigma}^{q} \sigma\right)$ 
experienced by the light $(u, d)$ quark (antiquark) constituents in the medium. There are additional negligible contributions to the mass shift of the hadron due to the center of mass and gluonic fluctuation effects taken into account by a parameter, $z_{h}$ in the QMC model [8, 9]. The mass shifts of the hadrons with the same light $(q=u, d)$ quark (antiquark) constituents e.g., for $K(\bar{K})$ and $K^{*}\left(\bar{K}^{*}\right)$ mesons, are thus observed to be very close to each other, in spite of the fact that $K$ and $\bar{K}$ are pesudoscalar mesons with spin zero, whereas $K^{*}$ and $\bar{K}^{*}$ mesons have spin, $S=1$. It might, however, be mentioned here that in order to reproduce the empirically extracted replusive $K^{+} N$ total potential from the repulsive $K^{+}$-Nucleus interaction [8], the vector potential $V_{\omega}^{q}$ of the constituent light quark (antiquark) of $K(\bar{K})$ has to be increased by a factor of $(1.4)^{2}$ in the QMC model [8]. Within the framework of the coupled channel approach, the resonance $\Lambda(1405)$ is generated dynamically from the $\bar{K} N$ interaction using the lowest order Weinberg-Tomozawa interaction in a chiral Lagrangian [10]. The $\bar{K} N$ scattering amplitude in the hadronic medium is obtained by the self-consistent solution of the coupled channel Lippmann Schwinger equations, including the medium effects from the Pauli blocking, the mean field binding potentials for the baryons and the sel-energies of $\bar{K}$ and $\pi$ [11. For the $K N$ interaction, due to absence of any resonance near to the threshold, the $T \rho$ approximation is a good approximation for the study the self-energies of the kaons at low densities, and, a self-consistent calculation of the scattering amplitude has only small modifications to the results obtained using this approximation. Using the Parton-HadronString-Dynamics (PHSD) transport model, and, the in-medium spectral functions of the pseudoscalar $(K, \bar{K})$ mesons, as well as, of the vector $\left(K^{*}\right.$ and $\left.\bar{K}^{*}\right)$ mesons, calculated using the self-consistent coupled channel approach, the dynamics of $K^{*}$ and $\bar{K}^{*}$ in heavy ion collision experiments have been studied in Refs. [12, 13]. These calculations show that the production of the strange vector $K^{*}\left(\bar{K}^{*}\right)$ mesons by hadronization from the QGP phase is quite small at RHIC as well as at LHC and these vector mesons are dominantly produced at the later hadronic stage from $K(\bar{K}) \pi$ scatterings. In Ref. [13], the PHSD calculations have also been performed for heavy ion collision experiments at low beam energies relevant for the FAIR and NICA conditions, where the medium effects may become visible due to the large baryon density matter which will be created at these facilities.

In the present work, we study the relativstic Breit-Wigner spectral functions as well as production cross-sections for the vector mesons, $K^{*}, \bar{K}^{*}$ and $\phi$ mesons in isospin asymmetric strange hadronic matter. These are obtained from the mass modifications of the open strange 
mesons and the in-medium decay widths for the processes $\phi \rightarrow K \bar{K}, K^{*} \rightarrow K \pi$ and $\bar{K}^{*} \rightarrow$ $\bar{K} \pi$ calculated from the mass modifications of the $K(\bar{K})$ and $K^{*}\left(\bar{K}^{*}\right)$ mesons. The masses of the pseudocalar open strange mesons (kaons and antikaons) in the hadronic medium [14-17], have been calculated using a chiral SU(3) model [18, 19]. The mass modifications of the $K$ and $\bar{K}$ mesons in the isospin asymmetric hyperonic matter result from their interactions with the baryons and the scalar mesons. In the chiral SU(3) model, the interactions of the kaons and antikaons are due to the vectorial interaction with the baryons given by the WeinbergTomozawa term at the leading order, as well as, the scalar exchange and the range terms at the next to leading order in the chiral perturbation theory. The $K^{*}\left(\bar{K}^{*}\right)$ and the $K(\bar{K})$ mesons are observed to have very similar mass modifications in the medium in the QMC model, due to the identical light quark (and antiquark) constituents, i.e., $q \bar{s}(s \bar{q}), q=(u, d)$, of these mesons [8, 9]. In the present work, we assume the mass shifts of the vector $K^{*}$ and $\bar{K}^{*}$ mesons to be the same as the shifts in the masses of the pseudoscalar mesons $K$ and $\bar{K}$ mesons, the latter being calculated using the chiral SU(3) model [17]. The spectral functions as well as the production cross-sections of the vector mesons $\phi, K^{*}$ and $\bar{K}^{*}$ in the strange hadronic medium computed in the present work are observed to have significant modifications due to isospin asymmetry as well as strangeness fraction at high densities. These should have observable consequences on the production of the hidden and open strange mesons [1] in asymmetric heavy ion collisions at the compressed baryonic matter (CBM) experiments planned at the future facility at GSI.

The outline of the paper is as follows: In subsection IIA, we discuss briefly the study of the in-medium masses of the kaons and antikaons in isospin asymmetric strange hadronic matter within a chiral $\mathrm{SU}(3)$ model. The in-medium decay widths of the $\phi$ meson to $K \bar{K}$ and of the $K^{*}\left(\bar{K}^{*}\right)$ meson to $K(\bar{K}) \pi$, are calculated from the mass modifications of the open strange mesons. These decay widths are calculated using a field theoretical model of composite hadrons with quark (and antiquark) constituents as described in subsection IIB. Subsection IIC gives a description of the relativistic Breit-Wigner spectral functions as well as the production cross-sections of the vector mesons, $K^{*}, \bar{K}^{*}$ and $\phi$ as studied in the present work. In section III, we discuss the effects of isospin asymmetry, strangeness and density on the spectral properties and the production cross-sections of the strange vector mesons. Section IV summarizes the findings of the present investigation. 


\section{STRANGE MESONS IN ASYMMETRIC HYPERONIC MATTER}

\section{A. MASSES:}

The isospin asymmetric strange hadronic matter is described by a chiral SU(3) model based on a nonlinear realization of the chiral symmetry [20 22] and the broken scale invariance [23, 24]. The model describes the interactions of the baryons and the mesons (scalar, vector, pseudoscalar and axialvector) and the dilaton field, $\chi$, which is incorporated in the model to mimick the scale symmetry breaking of Quantum Chromodynamics (QCD). The model has been described in detail in Refs. [18, 19]. The general form of the Lagrangian density is given as [18]

$$
\mathcal{L}=\mathcal{L}_{\text {kin }}+\sum_{W} \mathcal{L}_{B W}+\mathcal{L}_{\text {vec }}+\mathcal{L}_{0}+\mathcal{L}_{\text {scalebreak }}+\mathcal{L}_{S B}
$$

where, $\mathcal{L}_{\text {kin }}$ corresponds to the kinetic energy terms of the baryons and the mesons, $\mathcal{L}_{B W}$ contains the baryon-meson interactions, $\mathcal{L}_{v e c}$ describes the dynamical mass generation of the vector mesons via couplings to the scalar fields and contains additionally quartic selfinteractions of the vector fields, $\mathcal{L}_{0}$ contains the meson-meson interaction terms $\mathcal{L}_{\text {scalebreak }}$ is a scale invariance breaking logarithmic potential, $\mathcal{L}_{S B}$ describes the explicit chiral symmetry breaking. The kinetic energy terms are given as

$$
\begin{aligned}
\mathcal{L}_{k i n} & =i \operatorname{Tr} \bar{B} \gamma_{\mu} D^{\mu} B+\frac{1}{2} \operatorname{Tr} D_{\mu} X D^{\mu} X+\operatorname{Tr}\left(u_{\mu} X u^{\mu} X+X u_{\mu} u^{\mu} X\right)+\frac{1}{2} \operatorname{Tr} D_{\mu} Y D^{\mu} Y \\
& +\frac{1}{2} D_{\mu} \chi D^{\mu} \chi-\frac{1}{4} \operatorname{Tr}\left(V_{\mu \nu} V^{\mu \nu}\right)-\frac{1}{4} \operatorname{Tr}\left(\mathcal{A}_{\mu \nu} \mathcal{A}^{\mu \nu}\right)-\frac{1}{4} \operatorname{Tr}\left(F_{\mu \nu} F^{\mu \nu}\right),
\end{aligned}
$$

where, $B$ is the baryon octet, $X$ is the scalar meson multiplet, $Y$ is the pseudoscalar chiral singlet, $\chi$ is the scalar dilaton field, $V_{\mu \nu}=\partial_{\mu} V_{\nu}-\partial_{\nu} V_{\mu}, \mathcal{A}_{\mu \nu}=\partial_{\mu} \mathcal{A}_{\nu}-\partial_{\nu} \mathcal{A}_{\mu}$, and $F_{\mu \nu}$ are the field strength tensors of the vector meson multiplet, $V^{\mu}$, the axial vector meson multiplet $\mathcal{A}^{\mu}$ and the photon field, $A^{\mu}$. In equation $(2), u_{\mu}=-\frac{i}{4}\left[\left(u^{\dagger}\left(\partial_{\mu} u\right)-\left(\partial_{\mu} u^{\dagger}\right) u\right)-\left(u\left(\partial_{\mu} u^{\dagger}\right)-\left(\partial_{\mu} u\right) u^{\dagger}\right)\right]$, where, $u=\exp \left[\frac{i}{\sigma_{0}} \pi^{a} \lambda^{a} \gamma_{5}\right]$. The covariant derivative of a field $\Phi(\equiv B, X, Y, \chi)$ reads $D_{\mu} \Phi=\partial_{\mu} \Phi+\left[\Gamma_{\mu}, \Phi\right]$, with $\Gamma_{\mu}=-\frac{i}{4}\left[\left(u^{\dagger}\left(\partial_{\mu} u\right)-\left(\partial_{\mu} u^{\dagger}\right) u\right)+\left(u\left(\partial_{\mu} u^{\dagger}\right)-\left(\partial_{\mu} u\right) u^{\dagger}\right)\right]$.

$\mathcal{L}_{B W}$ in equation (1) describes the interactions of the baryons with the meson, $W$ and has the general form [18]

$$
\mathcal{L}_{\mathcal{B W}}=\sqrt{2} g_{8}^{W}\left(\alpha_{W}[\bar{B} O B W]_{F}+\left(1-\alpha_{W}\right)[\bar{B} O B W]_{D}\right)-g_{1}^{W} \frac{1}{\sqrt{3}} \operatorname{Tr}(\bar{B} O B) \operatorname{Tr} W
$$


with $[\bar{B} O B W]_{F}=\operatorname{Tr}(\bar{B} O W B-\bar{B} O B W)$ and $[\bar{B} O B W]_{D}=\operatorname{Tr}(\bar{B} O W B+\bar{B} O B W)-$ $\frac{2}{3} \operatorname{Tr}(\bar{B} O B) \operatorname{Tr} W$. The above Lagrangian describes the interactions of the baryons with scalar mesons $(W=X, O=1)$, with vector mesons $\left(W=V_{\mu}, O=\gamma^{\mu}\right)$, with axial vector mesons $\left(W=\mathcal{A}_{\mu}, O=\gamma^{\mu} \gamma^{5}\right)$ and with pseudoscalar mesons $\left(W=u_{\mu}, O=\gamma^{\mu} \gamma^{5}\right)$. The hadronic matter is described using the mean field approximation, which replaces the meson fields with their expectation values. Within this approximation, every scalar field, $\phi(x) \rightarrow\langle\phi\rangle \equiv \phi$ and vector field, $V^{\mu}(x) \rightarrow \delta^{\mu 0}\left\langle V^{\mu}\right\rangle \equiv \delta^{\mu 0} V^{0}$. Assuming the hadronic matter to be uniform, static as well as rotationally invariant system, the expectation values are independent of spacetime and $\left\langle V^{i}\right\rangle=0$ [7]. The expectation values of the isovector scalar and isovector vector meson fields, $\delta^{a}$ and $\rho^{\mu a}$ with $a=1,2,3$, are replaced by $\delta^{a} \delta^{a 3}$ and the $\rho^{\mu a} \delta^{\mu 0} \delta^{a 3}$, as there is contribution only from the neutral scalar and vector mesons for the ground state. The baryon-scalar meson interaction generate the masses of the baryons and the experimentally observed vacuum masses are fitted from the parameters $\alpha_{s}, g_{1}^{S}$ and $g_{8}^{S}$. In the mean field approximation, the baryon-meson interaction terms have contributions only from the scalar mesons and the vector mesons and are given as

$$
\mathcal{L}_{B S}+\mathcal{L}_{B V}=-\sum_{i} \bar{\psi}_{i}\left[m_{i}^{*}+g_{\omega i} \gamma^{0} \omega+g_{\rho i} \gamma^{0} \rho+g_{\phi i} \gamma^{0} \phi\right] \psi_{i}
$$

The baryon-scalar meson interaction term, $\mathcal{L}_{B S}$ generates the masses of the baryons in the octet. The effective mass of the $i$-th baryon $\left(i=p, n, \Sigma^{ \pm}, \Sigma^{0}, \Xi^{0}, \Xi^{-}\right)$, described by the fermion field $\psi_{i}$, is given as

$$
m_{i}^{*}=-\left(g_{\sigma i} \sigma+g_{\zeta i} \zeta+g_{\delta i} \delta\right),
$$

where $\sigma, \zeta$ and $\delta$ are the expectation values of the non-strange scalar isoscalar field, $\sigma$, strange scalar isoscalar field $\zeta$ and third component of the non-strange scalar isovector $\delta$ fields respectively. The fields $\sigma, \zeta$ and $\delta$ may be identified as the particles $f_{0}(500), f_{0}(980)$ and $a_{0}(980)$ of the Particle Data Group [25]. The expectation values of the meson fields are determined by solving the coupled equations of motion of these fields [19] in the mean field approximation, additionally assuming that

$$
\bar{\psi}_{i} \psi_{j} \rightarrow \delta_{i j}\left\langle\bar{\psi}_{i} \psi_{i}\right\rangle \equiv \delta_{i j} \rho_{i}^{s}, \quad \bar{\psi}_{i} \gamma^{\mu} \psi_{j} \rightarrow \delta_{i j} \delta^{\mu 0}\left\langle\bar{\psi}_{i} \gamma^{0} \psi_{i}\right\rangle \equiv \delta_{i j} \delta^{\mu 0} \rho_{i}
$$

where, $\rho_{i}^{s}$ and $\rho_{i}$ are the scalar and number densities of baryon of species $i$. These are calculated for given values of the isospin asymmetry parameter, $\eta=-\frac{\sum_{i} I_{3 i} \rho_{i}}{\rho_{B}}$, and the strangeness fraction, $f_{s}=\frac{\sum_{i} s_{i} \rho_{i}}{\rho_{B}}$, where $I_{3 i}$ is the third component of the isospin of the 
baryon of species, $i$ and $s_{i}$ is the number of strange quarks in the $i$-th baryon. In the isospin asymmetric hadronic medium, the expectation value of the third component of the scalarisoscalar field $\delta(\sim(\bar{u} u-\bar{d} d))$ becomes non-zero, and leads to the masses of the baryons (given by equation (5) ) within a given isospin multiplet, to be different, determined by $g_{\delta i}$, the coupling of the $i$-th baryon with the field, $\delta$.

The modifications of the masses of the kaon and antikaon in the isospin asymmetric strange hadronic matter are calculated from the interactions of these mesons with the baryons and the scalar mesons in the chiral SU(3) model. These interactions include the Weinberg-Tomozawa term at the leading order, as well as the scalar exchange and the range terms in the next to leading order of chiral perturbation theory. The dispersion relations of the $K$ and $\bar{K}$ mesons are obtained by Fourier transformations of the equations of motion of these mesons [17]. These are given as

$$
-\omega^{2}+|\mathbf{k}|^{2}+m_{K}^{2}-\Pi_{K(\bar{K})}(\omega,|\mathbf{k}|)=0,
$$

where $\Pi(\omega,|\mathbf{k}|)$ denotes the kaon (antikaon) self energy in the medium. Explicitly, the self energy $\Pi(\omega,|\mathbf{k}|)$ for the kaon doublet, $\left(K^{+}, K^{0}\right)$ is given as

$$
\begin{aligned}
\Pi_{K}(\omega,|\mathbf{k}|) & =-\frac{1}{4 f_{K}^{2}}\left[3\left(\rho_{p}+\rho_{n}\right) \pm\left(\rho_{p}-\rho_{n}\right) \pm 2\left(\rho_{\Sigma^{+}}-\rho_{\Sigma^{-}}\right)\right. \\
& \left.-\left(3\left(\rho_{\Xi^{-}}+\rho_{\Xi^{0}}\right) \pm\left(\rho_{\Xi^{-}}-\rho_{\Xi^{0}}\right)\right)\right] \omega \\
& +\frac{m_{K}^{2}}{2 f_{K}}\left(\sigma^{\prime}+\sqrt{2} \zeta^{\prime} \pm \delta^{\prime}\right) \\
& +\left[-\frac{1}{f_{K}}\left(\sigma^{\prime}+\sqrt{2} \zeta^{\prime} \pm \delta^{\prime}\right)+\frac{d_{1}}{2 f_{K}^{2}}\left(\rho_{p}^{s}+\rho_{n}^{s}\right.\right. \\
& \left.+\rho_{\Lambda^{0}}^{s}+\rho_{\Sigma^{+}}^{s}+\rho_{\Sigma^{0}}^{s}+\rho_{\Sigma^{-}}^{s}+\rho_{\Xi^{-}}^{s}+\rho_{\Xi^{0}}^{s}\right) \\
& +\frac{d_{2}}{4 f_{K}^{2}}\left(\left(\rho_{p}^{s}+\rho_{n}^{s}\right) \pm\left(\rho_{p}^{s}-\rho_{n}^{s}\right)+\rho_{\Sigma^{0}}^{s}+\frac{5}{3} \rho_{\Lambda^{0}}^{s}\right. \\
& \left.\left.+\left(\rho_{\Sigma^{+}}^{s}+\rho_{\Sigma^{-}}^{s}\right) \pm\left(\rho_{\Sigma^{+}}^{s}-\rho_{\Sigma^{-}}^{s}\right)+2 \rho_{\Xi^{-}}^{s}+2 \rho_{\Xi^{0}}^{s}\right)\right]\left(\omega^{2}-|\mathbf{k}|^{2}\right)
\end{aligned}
$$

where the \pm signs refer to the $K^{+}$and $K^{0}$ respectively. In the above, $\sigma^{\prime}\left(=\sigma-\sigma_{0}\right), \zeta^{\prime}(=$ $\left.\zeta-\zeta_{0}\right)$ and $\delta^{\prime}\left(=\delta-\delta_{0}\right)$ are the fluctuations of the scalar-isoscalar fields $\sigma$ and $\zeta$, and the third component of the scalar-isovector field, $\delta$, from their vacuum expectation values. The vacuum expectation value of $\delta$ is zero $\left(\delta_{0}=0\right)$, since a nonzero value for it will break the isospin symmetry of the vacuum. The fluctuation of the dilaton field in the medium has been neglected in the present work, as it is related to the gluon condensate, whose modification in the hadronic medium is negligible. 
Similarly, for the antikaon doublet, $\left(K^{-}, \bar{K}^{0}\right)$, the self energy is calculated as [17]

$$
\begin{aligned}
\Pi_{\bar{K}}(\omega,|\mathbf{k}|)= & \frac{1}{4 f_{K}^{2}}\left[3\left(\rho_{p}+\rho_{n}\right) \pm\left(\rho_{p}-\rho_{n}\right) \pm 2\left(\rho_{\Sigma^{+}}-\rho_{\Sigma^{-}}\right)\right. \\
& \left.-\left(3\left(\rho_{\Xi^{-}}+\rho_{\Xi^{0}}\right) \pm\left(\rho_{\Xi^{-}}-\rho_{\Xi^{0}}\right)\right)\right] \omega \\
& +\frac{m_{K}^{2}}{2 f_{K}}\left(\sigma^{\prime}+\sqrt{2} \zeta^{\prime} \pm \delta^{\prime}\right) \\
& +\left[-\frac{1}{f_{K}}\left(\sigma^{\prime}+\sqrt{2} \zeta^{\prime} \pm \delta^{\prime}\right)+\frac{d_{1}}{2 f_{K}^{2}}\left(\rho_{p}^{s}+\rho_{n}^{s}\right.\right. \\
& \left.+\rho_{\Lambda^{0}}^{s}+\rho_{\Sigma^{+}}^{s}+\rho_{\Sigma^{0}}^{s}+\rho_{\Sigma^{-}}^{s}+\rho_{\Xi^{-}}^{s}+\rho_{\Xi^{0}}^{s}\right) \\
& +\frac{d_{2}}{4 f_{K}^{2}}\left(\left(\rho_{p}^{s}+\rho_{n}^{s}\right) \pm\left(\rho_{p}^{s}-\rho_{n}^{s}\right)+\rho_{\Sigma^{0}}^{s}+\frac{5}{3} \rho_{\Lambda^{0}}^{s}\right. \\
& \left.\left.+\left(\rho_{\Sigma^{+}}^{s}+\rho_{\Sigma^{-}}^{s}\right) \pm\left(\rho_{\Sigma^{+}}^{s}-\rho_{\Sigma^{-}}^{s}\right)+2 \rho_{\Xi^{-}}^{s}+2 \rho_{\Xi^{0}}^{s}\right)\right]\left(\omega^{2}-|\mathbf{k}|^{2}\right)
\end{aligned}
$$

where the \pm signs refer to the $K^{-}$and $\bar{K}^{0}$ respectively. In the equations (8) and (9), the first term is due to the vectorial Weinberg-Tomozawa term, the second term is the contribution due to the scalar exchange, and, the last three terms are due to the range terms. The parameters $d_{1}$ and $d_{2}$ of the range terms are determined to be $2.56 / m_{K}$ and $0.73 / m_{K}$, by a fit of the empirical values of the $\mathrm{KN}$ scattering lengths for $\mathrm{I}=0$ and $\mathrm{I}=1$ channels [1517], taken as $a_{K N}(I=0) \approx-0.09 \mathrm{fm}$ and $a_{K N}(I=1) \approx-0.31 \mathrm{fm}[26$ 28]. The masses of the kaons and antikaons in the medium, $m_{K}^{*}$ and $m_{\bar{K}}^{*}$ defined as the energies of these mesons for $|\mathbf{k}|=0$, are obtained from the solution of the dispersion relation (7) for $|\mathbf{k}|=0$ [1]. In the early work based on chiral perturbation theory [4, the masses of the kaons and antikaons in the nuclear medium were obtained by solving the dispersion relation for $|\mathbf{k}|=0$, using the leading order vectorial Weinberg-Tomozawa term and the attractive $K N$ sigma term at the sub-leading order, the latter largely responsible for the possibility of anikaon condensation in the interior of neutron stars due to drop of the mass of the antikaons in nuclear matter. The Weinberg-Tomozawa term leads to an attraction (repulsion) for the kaons (antikaons) in nuclear matter. In the chiral effective model used in the present work, the mass modifications of the kaons and antikaons arise from their interactions with the baryons and the scalar mesons retaining the leading order vectorial Weinberg-Tomozawa interaction, as well as, the scalar exchange and the range terms at the next to leading order. For the isospin symmetric hadronic matter $(\eta=0)$, as has already been mentioned, $\delta=0$, the scalar fields $\sigma$ and $\zeta$ are obtained from their equations of motion. For the isospin asymmetric hadronic medium, $(\eta \neq 0)$, the expectation values of the scalar fields are obtained by solving 
the coupled equations for $\sigma, \zeta$ and $\delta$, which are used in the dispersion relation given by (7) to obtain the in-medium masses of the $K$ and $\bar{K}$ mesons for given values of the baryon density, $\rho_{B}$, the isospin asymmetry parameter, $\eta$ and the strangeness fraction, $f_{s}$.

The in-medium decay widths of $\phi \rightarrow K \bar{K}$ and $K^{*}\left(\bar{K}^{*}\right) \rightarrow K(\bar{K}) \pi$ are calculated from the mass modifications of the open strange mesons. The in-medium masses of the kaons and the antikaons have been calculated in isospin asymmetric (nuclear) hyperonic matter, using the chiral SU(3) model in Ref. [17]. It might be noted here that the interactions of the open strange vector mesons, $K^{*}$ and $\bar{K}^{*}$ with the baryons, using the Lagrangian $\mathcal{L}_{B W}$, for $W=V$, turn out to be of the form $\bar{\psi}^{i} \gamma^{\mu} \psi^{j} V_{\mu}$, with $i \neq j$, which vanish due to the

assumption $\bar{\psi}_{i} \gamma^{\mu} \psi_{j} \rightarrow \delta_{i j}{ }^{\mu 0}\left\langle\bar{\psi}_{i} \gamma^{0} \psi_{i}\right\rangle \equiv \delta_{i j}{ }^{\mu 0} \rho_{i}$, as given by equation (6). The only vector meson fields which have non-zero contributions from the baryon-meson interaction terms given by equation (3) are the $\omega, \rho_{0}$ and $\phi$ mesons. The calculation of the mass modifications of the $K^{*}$ and $\bar{K}^{*}$ mesons using the chiral effective model is thus beyond the scope of the present work. As has already been mentioned, in the present work, we assume the mass modification of the vector open strange meson, $K^{*}\left(\bar{K}^{*}\right)$ to be same as for the $K(\bar{K})$ meson, i.e, $\Delta m_{K^{*}\left(\bar{K}^{*}\right)} \equiv m_{K^{*}\left(\bar{K}^{*}\right)}^{*}-m_{K^{*}\left(\bar{K}^{*}\right)}^{v a c}=m_{K(\bar{K})}^{*}-m_{K(\bar{K})}^{v a c}$. The decay widths of $\phi \rightarrow K \bar{K}$, $K^{*}\left(\bar{K}^{*}\right) \rightarrow K(\bar{K}) \pi$ are calculated using a field theoretical model of hadrons with quark (and antiquark) constituents as described in the following subsection.

\section{B. DECAY WIDTHS:}

The decay widths of $\phi \rightarrow K \bar{K}$ and $K^{*}\left(\bar{K}^{*}\right) \rightarrow K(\bar{K}) \pi$ are calculated using a field theoretic model of composite hadrons [29, 30]. These are evaluated from the matrix element of the light quark-antiquark pair creation term of the free Dirac Hamiltonian between initial and final states [31 35]. For a generic decay process $A \rightarrow B+C$, the matrix element is multiplied with a parameter $\gamma_{A}$, which is a measure of the light quark-antiquark pair creation strength to form the final mesons $B$ and $C$ from the decay of the parent meson $A$. This parameter is fitted from the observed decay width of $A \rightarrow B+C$ in vacuum. This is similar to the calculation of the decay width of $A \rightarrow B+C$ in the ${ }^{3} P_{0}$ model [36, 37], where the decay of the meson $A$ at rest to mesons $B$ and $C$ proceeds with a light quark-antiquark pair creation in the ${ }^{3} P_{0}$ state, and, the matrix element is multiplied by a parameter, which is a measure of the quark-antiquark pair creation strength, fitted from the measured value of 

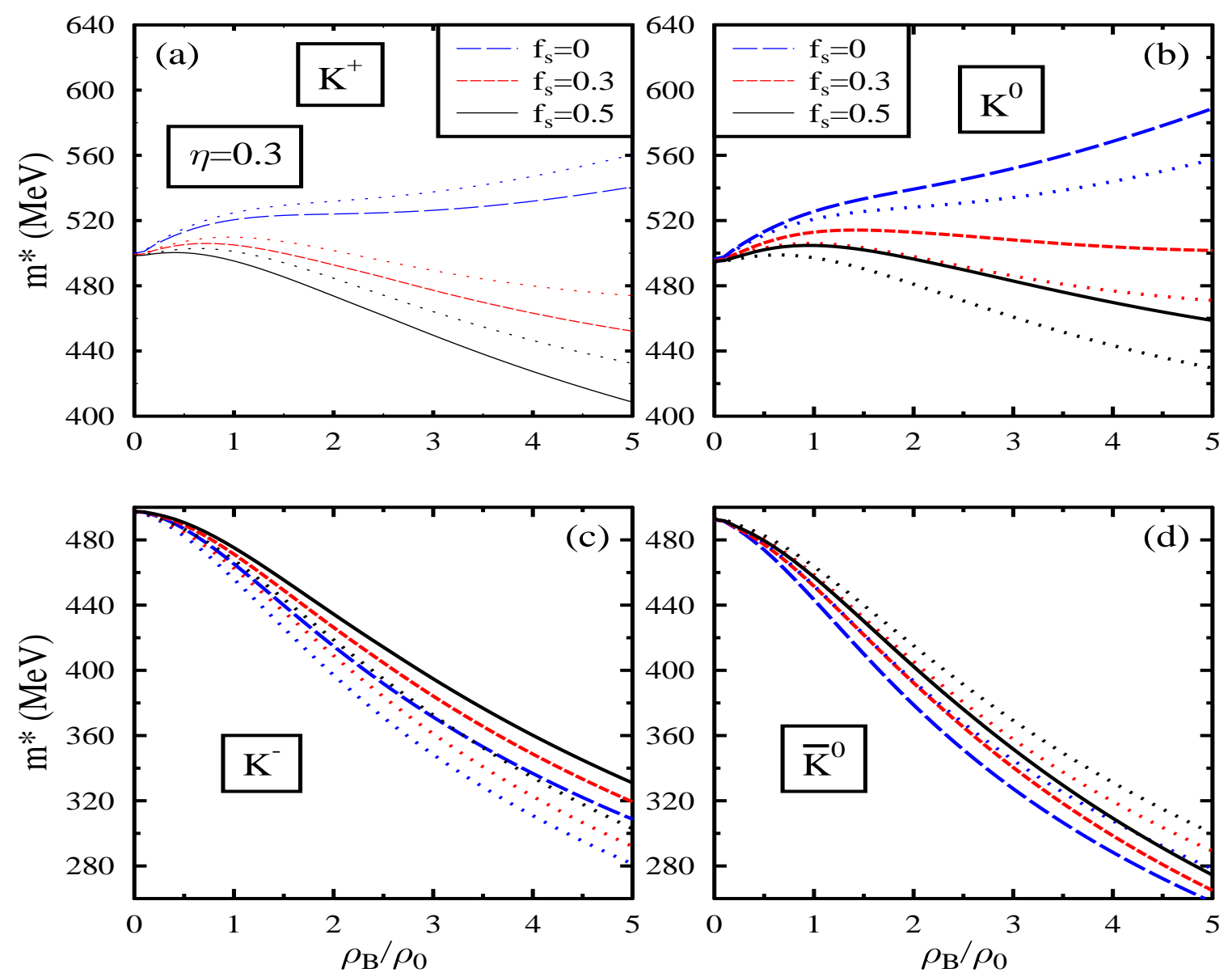

FIG. 1: The masses of $K\left(K^{+}, K^{0}\right)$ and $\bar{K}\left(K^{-}, \bar{K}^{0}\right)$ mesons in the (strange) hadronic matter with strangenss fractions, $f_{s}=0,0.3,0.5$ for isospin asymmetric parameter $\eta=0.3$. These are compared with the isospin symmetric case $(\eta=0)$ shown as dotted lines. 

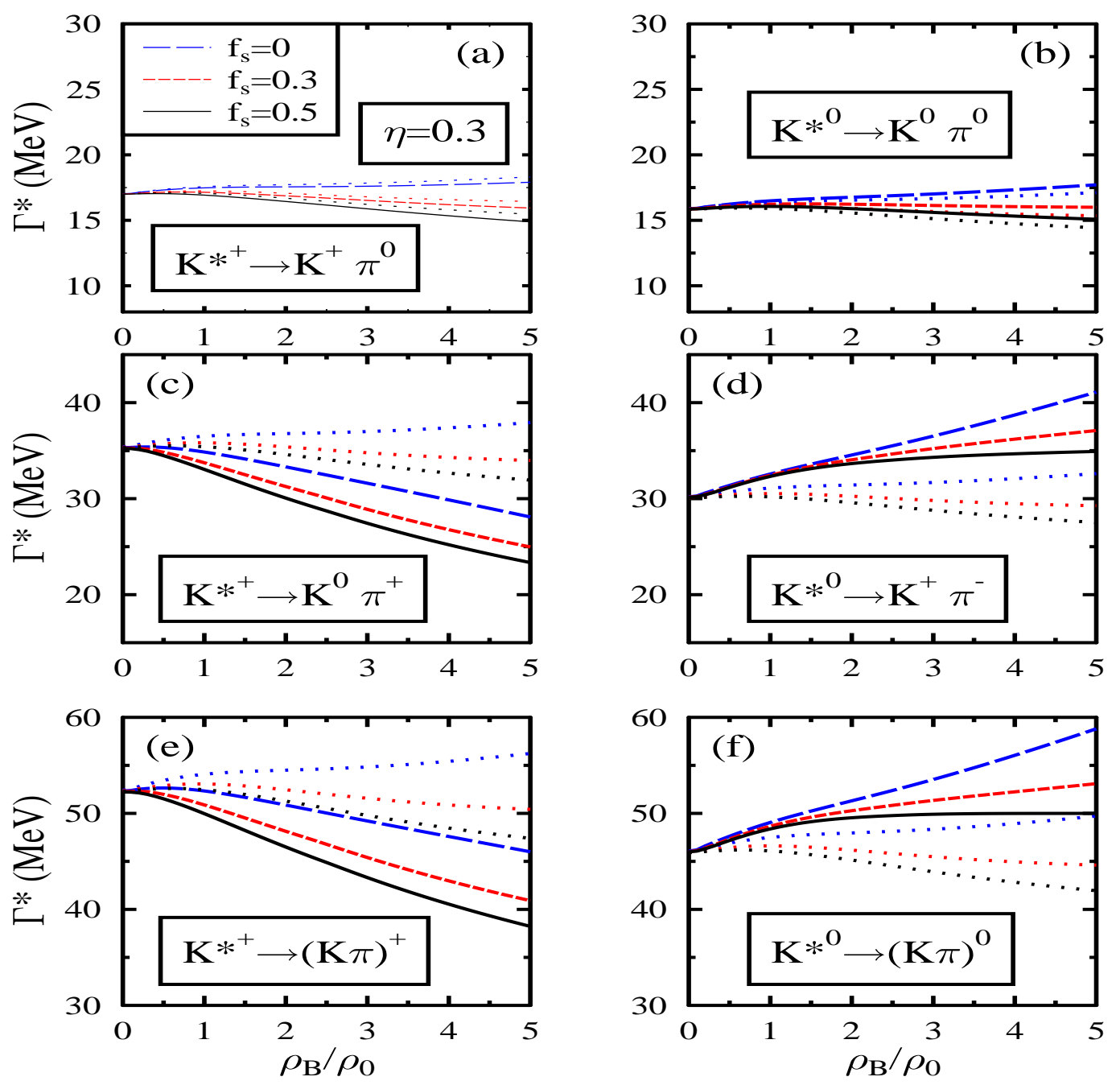

FIG. 2: Decay widths of the charged open strange vector meson, $K^{*}\left(K^{*+}, K^{* 0}\right)$ to $(K \pi)^{+, 0}$ for $f_{s}=0,0.3,0.5$ for isospin asymmetric matter with $\eta=0.3$. These are compared to the results obtained for symmetric matter $(\eta=0)$ shown as dotted lines. 

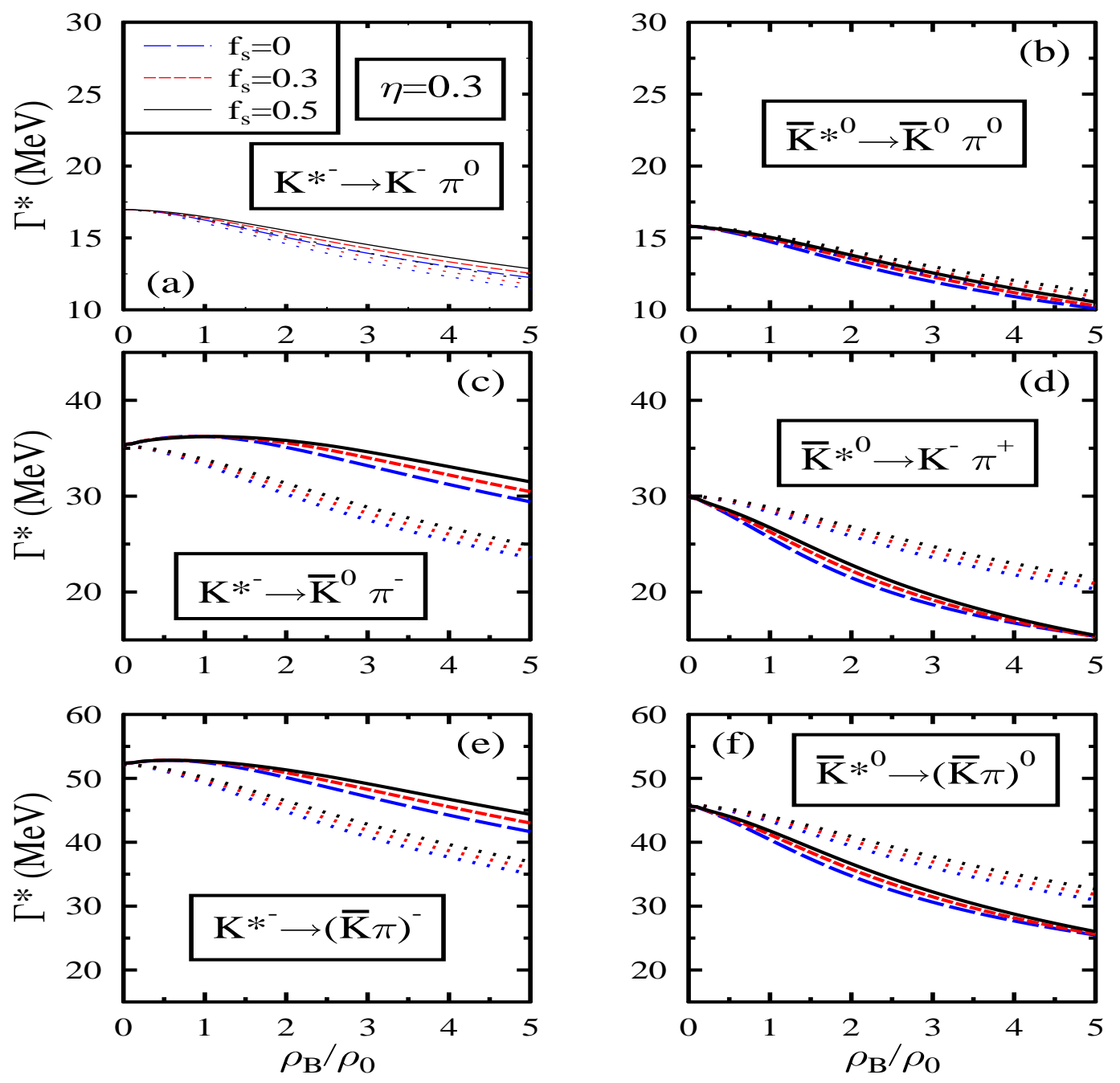

FIG. 3: Decay widths of the charged open strange vector meson, $\bar{K}^{*}\left(K^{*-}, \overline{K^{* 0}}\right)$ to $(\bar{K} \pi)^{-, 0}$ for $f_{s}=0,0.3,0.5$ for isospin asymmetric matter with $\eta=0.3$. These are compared to the results obtained for symmetric matter $(\eta=0)$ shown as dotted lines. 

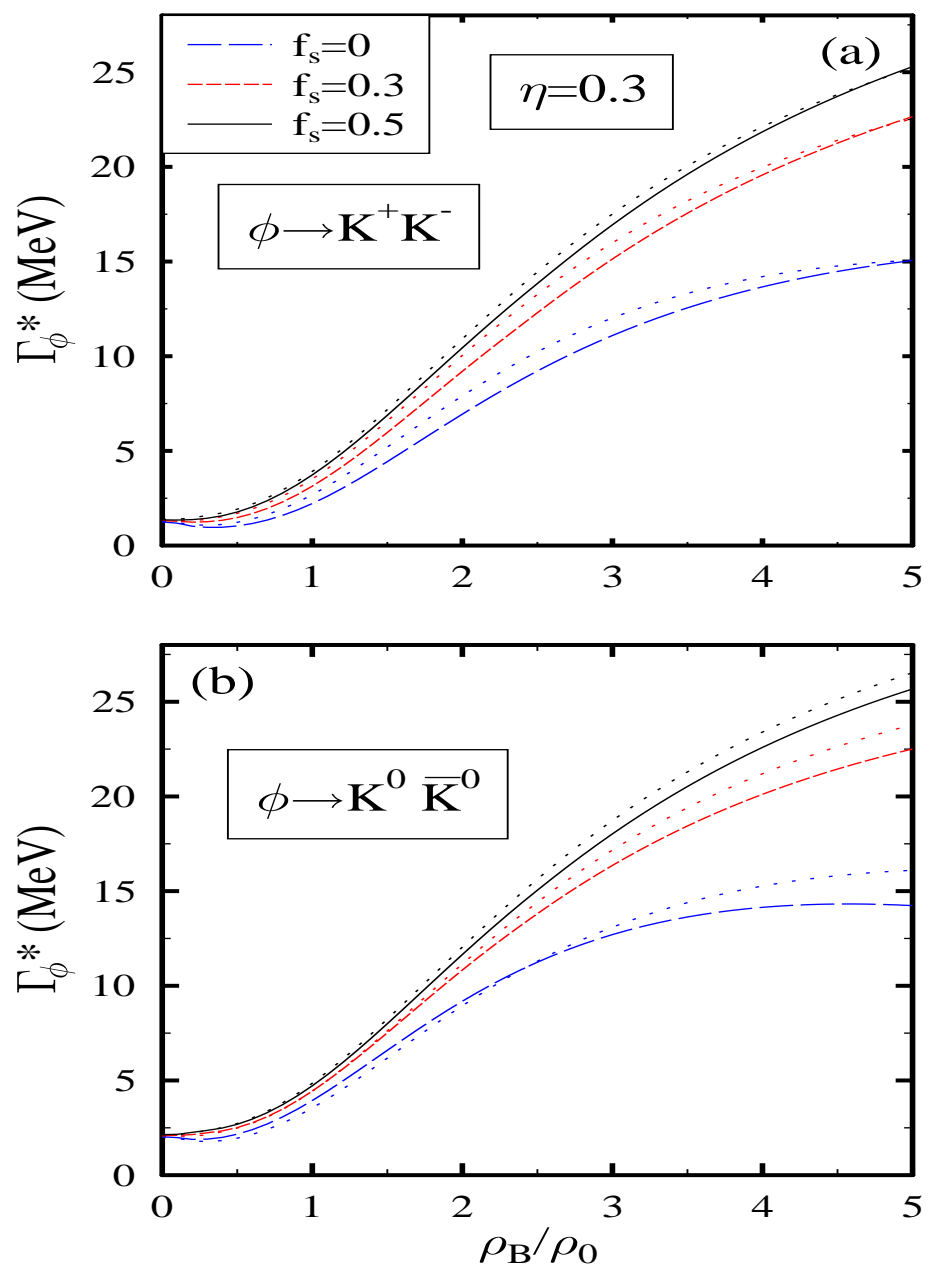

FIG. 4: Decay widths of $\phi$ to charged and neutral $K \bar{K}$ in strange hadronic matter, for $f_{s}=$ $0,0.3,0.5$ for $\eta=0.3$. These are compared with the results for isospin symmetric matter shown as dotted lines. 
the decay width in vacuum. The ${ }^{3} P_{0}$ model has been extensively used to study the decay processes of the mesons [36 38] in vacuum. In the charm sector, the model is observed to explain the experimentally observed strong suppression of the decay modes of the $\psi(4040)$ to $D \bar{D}$ as well as $\left(D \bar{D}^{*}+\bar{D} D^{*}\right)$, as compared to the final state $D^{*} \bar{D}^{*}$ in vaccum [39]. This suggests the importance of accounting for the internal structure of the hadrons, i.e., the quark (and antiquark) constituents, to study their decay widths. The ${ }^{3} P_{0}$ model has been used for the study of the in-medium decay widths of the charmonium state to $D$ and $\bar{D}$ mesons from the mass modifications of the $D$ and $\bar{D}$ mesons [40] as well as from the inmedium masses of the charmonium as well as open charm mesons calculated using a chiral effective model [41]. The process $\Psi \rightarrow D \bar{D}$ proceeds with $c(\bar{c})$ of the charmonium state, $\Psi$ combining with the light antiquark (quark) of the quark-antiquark created in the ${ }^{3} P_{0}$ state, to form the $D$ and $\bar{D}$ mesons in the final state. Taking the internal structure of the mesons in the initial and final states into account, along with the light constituent quark-antiquark pair creation into account, both the models, e.g., the ${ }^{3} P_{0}$ model as well as the model of composite hadron as considered in the present work, are observed to lead to vanishing of the decay widths at specific densities [31, 40, 41].

In the field theoretical model of composite hadrons considered in the present work, we study the medium modifications of the decay widths of $\phi \rightarrow K \bar{K}$ and $K^{*}\left(\bar{K}^{*}\right) \rightarrow K(\bar{K}) \pi$ from the mass modifications of the open strange mesons. These decay widths are calculated from the matrix element of the free Dirac Hamiltonian between the initial and final states, assuming the harmonic oscillator wave functions in the explicit constructions for these mesons [35]. The mesons $K, \bar{K}, K^{*}, \bar{K}^{*}, \pi$ and $\phi$ are assumed to be in the $1 \mathrm{~S}$ state The model has been used to study the decay widths of charmonium state to $D \bar{D}$ as well as $D^{*} \rightarrow D \pi$ [31] and of bottomonium states to $B \bar{B}$ in isospin asymmetric strange hadronic matter [32. The effect of strong magnetic fields has also been considered on the charmonium decay widths to $D \bar{D}$ [33], $D^{*} \rightarrow D \pi$ [34] as well as $K^{*} \rightarrow K \pi$ [35], where the pseudoscalar vector meson mixing effect is observed to have significant contributions to the masses of these mesons, in addition to the Landau level contributions to the masses of charged mesons.

The decay width of vector meson $A$ at rest decaying to pseudoscalar mesons $B(\mathbf{p})$ and $C(-\mathbf{p})$ is given as

$$
\Gamma(A \rightarrow B+C)=\gamma_{A}^{2} g^{2} \frac{8 \pi^{2} p_{B}^{0} p_{C}^{0}}{3 m_{A}} A_{A}(|\mathbf{p}|)^{2}|\mathbf{p}|^{3},
$$

where $p_{B}^{0}=\left(|\mathbf{p}|^{2}+m_{B}^{2}\right)^{1 / 2}$ and $p_{C}^{0}=\left(|\mathbf{p}|^{2}+m_{B}^{2}\right)^{1 / 2}$ are the energies of the outgoing $B$ and 

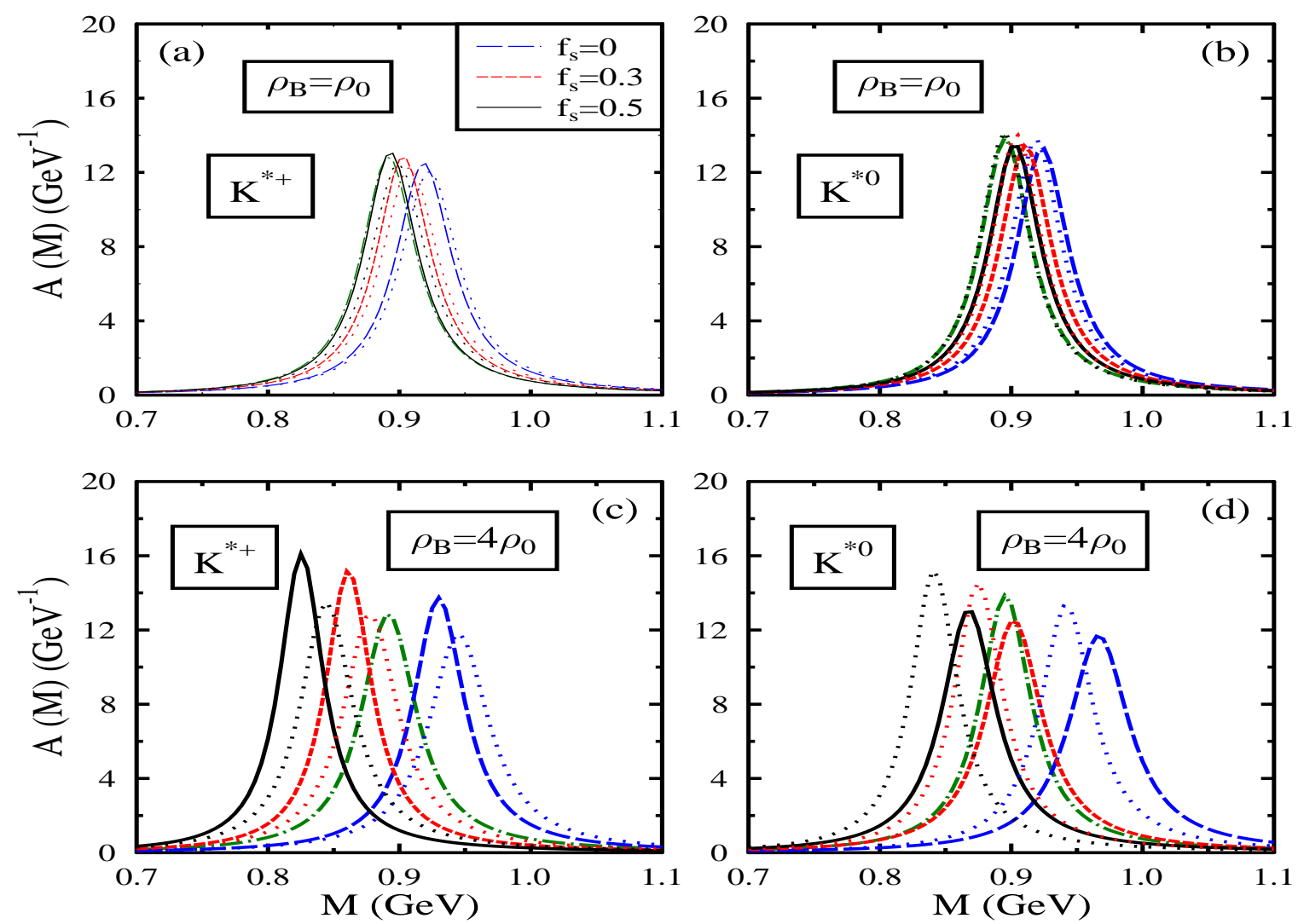

FIG. 5: Spectral functions of the $K^{*}\left(K^{*+}, K^{* 0}\right)$ mesons for $f_{s}=0,0.3,0.5$ for isospin asymmetric matter with $\eta=0.3$. These are compared with the results for isospin symmetric matter shown as dotted lines. The vacuum spectral function is shown as the dot-dashed line.

$C$ mesons respectively, in terms of the magnitude of the 3-momentum of $B(C)$ meson, $|\mathbf{p}|$, given as

$$
|\mathbf{p}|=\left(\frac{m_{A}^{2}}{4}-\frac{m_{B}^{2}+m_{C}^{2}}{2}+\frac{\left(m_{B}^{2}-m_{C}^{2}\right)^{2}}{4 m_{A}^{2}}\right)^{1 / 2}
$$



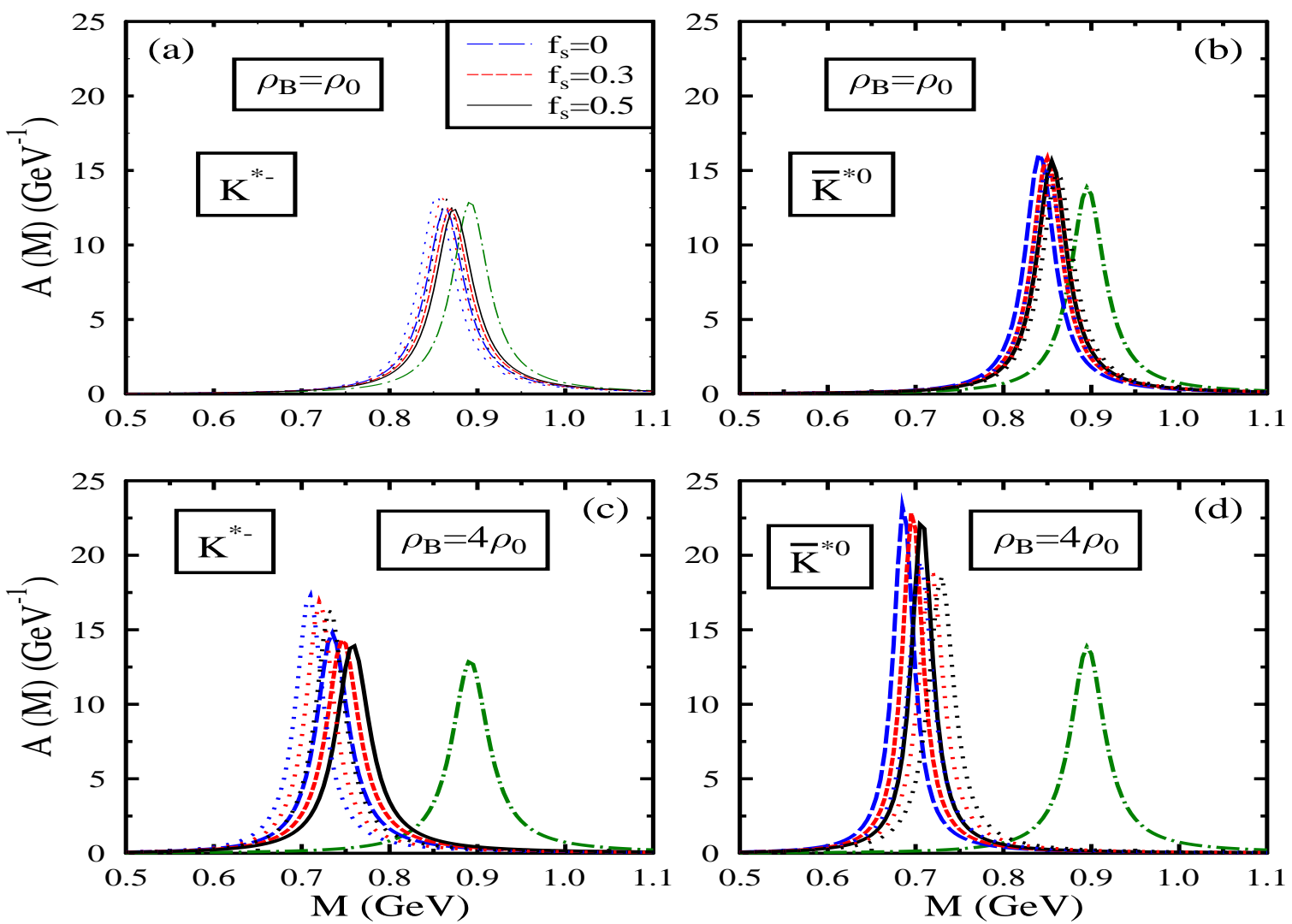

FIG. 6: Spectral functions of the $\bar{K}^{*}\left(K^{*-}, \overline{K^{* 0}}\right)$ mesons for $f_{s}=0,0.3,0.5$ for isospin asymmetric matter with $\eta=0.3$. These are compared with the results for isospin symmetric matter shown as dotted lines. The vacuum spectral function is shown as the dot-dashed line.

For the decay $K^{*}\left(\bar{K}^{*}\right) \rightarrow K(\bar{K}) \pi, A_{K^{*}}(|\mathbf{p}|)$ is given as

$$
A_{K^{*}}(|\mathbf{p}|)=6 c_{K^{*}}\left(\frac{\pi}{a_{K^{*}}}\right)^{3 / 2} \exp \left[\left(a_{K^{*}} b_{K^{*}}^{2}-\frac{1}{2}\left(\lambda_{2}^{2} R_{K}^{2}+\frac{1}{4} R_{\pi}^{2}\right)\right)|\mathbf{p}|^{2}\right]\left[F_{0 K^{*}}+\left(\frac{3 F_{1 K^{*}}}{2 a_{K^{*}}}\right)\right],
$$


where,

$$
\begin{aligned}
F_{0 K^{*}} & =\left(b_{K^{*}}-1\right)\left(1-\frac{1}{8 M_{q}^{2}}|\mathbf{p}|^{2}\left(\lambda_{2}-\frac{1}{2}\right)^{2}\right) \\
& -\left(b_{K^{*}}-\lambda_{2}\right)\left(\frac{1}{2}+\frac{1}{4 M_{q}^{2}}|\mathbf{p}|^{2}\left(\frac{3}{4} b_{K^{*}}^{2}-\frac{5}{4} b_{K^{*}}+\frac{7}{16}\right)\right) \\
& -\left(b_{K^{*}}-\frac{1}{2}\right)\left[\frac{1}{2}+\frac{1}{4 M_{q}^{2}}|\mathbf{p}|^{2}\left(\frac{3}{4} b_{K^{*}}^{2}-\left(1+\frac{1}{2} \lambda_{2}\right) b_{K^{*}}+\lambda_{2}-\frac{1}{4} \lambda_{2}^{2}\right)\right] \\
F_{1 K^{*}} & =-\frac{1}{4 M_{q}^{2}}\left[\frac{5}{2} b_{K^{*}}-\frac{9}{8}-\frac{11}{12} \lambda_{2}\right] .
\end{aligned}
$$

The parameters $a_{K^{*}}, b_{K^{*}}, c_{K^{*}}$ are given as

$a_{K^{*}}=\frac{\left(R_{K^{*}}^{2}+R_{K}^{2}+R_{\pi}^{2}\right)}{2}, \quad b_{K^{*}}=\frac{1}{2 a_{K^{*}}}\left(R_{K}^{2} \lambda_{2}+\frac{1}{2} R_{\pi}^{2}\right), \quad c_{K^{*}}=\frac{1}{12 \sqrt{3}}\left(\frac{R_{K^{*}}^{2} R_{K}^{2} R_{\pi}^{2}}{\pi^{3}}\right)^{3 / 4}$

In equations (13)-(14), $M_{q}$ is the constituent light quark $(q=u, d)$ mass. In equation (10), for the decay of $K^{*}\left(\bar{K}^{*}\right) \rightarrow K(\bar{K}) \pi$, the value of $g^{2}=1(2)$ for the neutral (charged) pion in the final state.

For the decay process $\phi \rightarrow K \bar{K}, A_{\phi}(|\mathbf{P}|)$ in equation 10$\rangle$ is given as

$$
A_{\phi}(|\mathbf{P}|)=6 c_{\phi}\left(\frac{\pi}{a_{\phi}}\right)^{3 / 2} \exp \left[\left(a_{\phi} b_{\phi}^{2}-R_{K}^{2} \lambda_{2}^{2}\right)|\mathbf{P}|^{2}\right]\left[F_{0 \phi}+F_{1 \phi} \frac{3}{2 a_{\phi}}\right]
$$

where $|\mathbf{P}|$ is the momentum of the outgoing $K(\bar{K})$ meson arising from the decay of $\phi$ meson at rest, which is given by equation (11), with particles $(\mathrm{A}, \mathrm{B}, \mathrm{C})$ given by $(\phi, K, \bar{K})$. In equation (16),

$$
\begin{aligned}
F_{0}^{\phi} & =\left(\lambda_{2}-1\right)-\frac{1}{2 M_{q}^{2}}|\mathbf{P}|^{2}\left(b_{\phi}-\lambda_{2}\right)\left(\frac{3}{4} b_{\phi}^{2}-\left(1+\frac{1}{2} \lambda_{2}\right) b_{\phi}+\lambda_{2}-\frac{1}{4} \lambda_{2}^{2}\right) \\
F_{1}^{\phi} & =\frac{1}{4 M_{q}^{2}}\left[-\frac{5}{2} b_{\phi}+\frac{2}{3}+\frac{11}{6} \lambda_{2}\right] .
\end{aligned}
$$

and the parameters $a_{\phi}, b_{\phi}$ and $c_{\phi}$ given as

$$
a_{\phi}=\frac{1}{2} R_{\phi}^{2}+R_{K}^{2}, \quad b_{\phi}=R_{K}^{2} \lambda_{2} / a_{\phi}, \quad c_{\phi}=\frac{1}{6 \sqrt{6}} \cdot\left(\frac{R_{\phi}^{2}}{\pi}\right)^{3 / 4} \cdot\left(\frac{R_{K}^{2}}{\pi}\right)^{3 / 2},
$$

\section{Spectral functions and Production Cross-sections of vector mesons}

The relativistic Breit Wigner spectral function of the vector meson $V$ is given as [12, 13, 42 


$$
A_{V}(M)=C_{1} \frac{2}{\pi} \frac{M^{2} \Gamma_{V}^{*}}{\left(M^{2}-m_{V}^{*}\right)^{2}+\left(M \Gamma_{V}^{*}\right)^{2}},
$$

where $M$ is the invariant mass and $C_{1}$ is a constant determined by normalization condition

$$
\int_{0}^{\infty} A_{V}(M) d M=1
$$

The in-medium relativistic Breit Wigner cross-section for the production of the vector meson, $V$ from the scattering of particles $a$ and $b$ is given as [12, 13, 43, 44]

$$
\sigma(M)=\frac{6 \pi^{2} \Gamma_{V}^{*} A_{V}(M)}{q\left(m_{V}^{*}, m_{a}^{*}, m_{b}^{*}\right)^{2}}
$$

In the above, $q\left(m_{V}^{*}, m_{a}^{*}, m_{b}^{*}\right)$ is the momentum of the scattering particle $a(b)$ in the center of mass frame of the vector meson, $V$, given as,

$$
q\left(m_{V}^{*}, m_{a}^{*}, m_{b}^{*}\right)=\frac{1}{2 M}\left(\left[m_{V}^{* 2}-\left(m_{a}^{*}+m_{b}^{*}\right)^{2}\right] \times\left[m_{V}^{* 2}-\left(m_{a}^{*}-m_{b}^{*}\right)^{2}\right]\right)^{1 / 2},
$$

with $m_{V}^{*}, m_{a}^{*}$ and $m_{b}^{*}$ as the in-medium masses of the vector meson, $V$ and the scattering particles $a$ and $b$ respectively. The vector meson, $V$ may, however, be created from scattering of (as well as decay to) particles in different modes, say particles $a_{i}$ and $b_{i}$ with masses $m_{a_{i}}^{*}$ and $m_{b_{i}}^{*}$ in the channel $i$. The in-medium decay width of the vector meson is then given as the sum of the decay widths in these channels, i.e., $\Gamma_{V}^{*}=\sum_{i} \Gamma_{V}^{*}{ }^{i}$. The production cross-section of the vector meson, accounting for all these channels is given as

$$
\sigma(M)=6 \pi^{2}\left(\sum_{i} \frac{\Gamma_{V}^{* i}}{q\left(m_{V}^{*}, m_{a_{i}}^{*}, m_{b_{i}}^{*}\right)^{2}}\right) A_{V}(M),
$$

where $q\left(m_{V}^{*}, m_{a_{i}}^{*}, m_{b_{i}}^{*}\right)$ is the center of mass momentum of the particle $a_{i}$ as well as $b_{i}$ corresponding to the channel $i$ in the center of mass frame of the vector meson, $V$.

In the present work, we study the in-medium spectral functions as well as the production cross-sections of the vector mesons, $K^{*}\left(K^{*+}, K^{* 0}\right), \bar{K}^{*}\left(K^{*-}, \bar{K}^{* 0}\right)$ and $\phi$ mesons in isospin asymmetric strange hadronic matter. For the processes $K^{*} \rightarrow K \pi, \bar{K}^{*} \rightarrow \bar{K} \pi$, and $\phi \rightarrow$ $K \bar{K}$, the in-medium decay widths of the vector mesons, $K^{*}, \bar{K}^{*}$ and $\phi$ are calculated from the medium modifications of the open strange mesons, using the field theoretic model of composite hadrons, as described in the previous subsection. The masses of the pseudoscalar strange mesons, $K$ and $\bar{K}$ mesons, as modified in the isospin asymmetric hyperonic matter are calculated using the chiral SU(3) model. The mass modifications of the $\phi$ meson and 
pions have not been taken into account in the present work. The shifts in the masses of the $K^{*}$ and $\bar{K}^{*}$ are assumed to be same as for the mass shifts of $K$ and $\bar{K}$ mesons [8, 9]. The in-medium spectral functions as well as the production cross-sections, given by equations (19) and 23) respectively, for the vector mesons, $K^{*}, \bar{K}^{*}$ and $\phi$ are investigated in the isospin asymmetric strange hadronic matter.

\section{RESULTS AND DISCUSSIONS}

The in-medium spectral functions for the hidden and open strange vector mesons $(\phi$, $K^{*}$ and $\left.\bar{K}^{*}\right)$ are investigated in the present work. The decay widths of the vector mesons for the decay processes $\phi \rightarrow K \bar{K}$ and $K^{*}\left(\bar{K}^{*}\right) \rightarrow K(\bar{K}) \pi$ are calculated using the field theoretic model of composite hadrons [35] as described in the previous section. The medium modifications of these decay widths are computed from the mass modifications of the open strange mesons in the hadronic medium. The masses of the kaons and antikaons in the isospin asymmetric strange hadronic medium have been calculated using a chiral $\mathrm{SU}(3)$ model in Ref. [17. The mass modifications of the $K$ and $\bar{K}$ mesons arise due to the vectorial Weinberg-Tomozawa term at the leading order as well as the scalar exchange and the range terms at the sub-leading level in the chiral perturbation theory. These are obtained by solving the dispersion relation given by equation (7) with the self-energies of the $K$ and $\bar{K}$ mesons given by equations (8) and (9) respectively. For the sake of completeness, the in-medium masses of the kaons and antikaons, as calculated in Ref. [17], are shown in figure 1. illustrating the effects due to the strangeness and isospin asymmetry of the hadronic medium.

For the isospin symmetric nuclear matter $\left(\eta=0, f_{s}=0\right)$, the Weinberg-Tomozawa term leads to a rise (drop) of the mass of the $K(\bar{K})$ meson. The scalar exchange term as well as the $d_{1}$ and $d_{2}$ range terms are attractive, whereas, the first range term is repulsive for both the kaons and antikaons. In the presence of isospin asymmetry in the medium $(\eta \neq 0)$, there are explicit contributions arising from the Weinberg-Tomozawa, the terms involving $\delta$ in the scalar exchange, the first range and the $d_{2}$ range terms. The $d_{1}$ range term also implicitly depends on the isospin asymmetry, where the values of the scalar densities of the baryons are obtained from the coupled equations of motion of the scalar fields, $\sigma, \zeta$ and $\delta$. For nonzero strangeness in the medium, there are contributions from the hyperons to the 
Weinberg-Tomozawa term and the $d_{1}$ and $d_{2}$ range terms.

As can be seen from figure 1, the isospin asymmetry of the medium is observed to lead to a drop (increase) in the mass of the $K^{+}\left(K^{0}\right)$ meson for the nuclear as well as in hyperonic matter and these effects are observed to be larger for the $K^{0}$ meson as compared to $K^{+}$ meson. In isospin symmetric nuclear matter $\left(\eta=0, f_{s}=0\right)$, the drop in the masses of the antikaons in the medium as shown in figure 1 is due to the attractive contributions from the Weinberg-Tomozawa term, scalar exchange as well as the $d_{1}$ and $d_{2}$ range terms, which dominate over the repulsive contribution from the first range term. There is observed to be a rise (drop) of the mass of the $K^{-}\left(\bar{K}^{0}\right)$ meson mass due to isospin asymmetry both in the nuclear as well as hyperonic matter. The effects of isospin asymmetry lead to mass differences in the $K^{+}$and $K^{0}$ as well as for $K^{-}$and $\bar{K}^{0}$ mesons (opposite in sign for the mass shifts of $K^{+}$and $K^{0}$ mesons in kaon doublet as well as of $K^{-}$and $\bar{K}^{0}$ mesons in the antikaon doublet). The mass shifts of around +27 and $-42 \mathrm{MeV}$ are obtained for $K$ and $\bar{K}$ mesons in isospin symmetric nuclear matter at the nuclear matter saturation density using the chiral $\mathrm{SU}(3)$ model [17]. These may be compared to the values of around +29 and $-50 \mathrm{MeV}$ [45] for these mesons calculated using a coupled channel approach [10]. In Ref. [10], using the lowest order chiral Lagrangian as well as a cut-off to regularize the loop integrals, the selfconsistent solutions of the coupled channel Lippmann-Schwinger equations for the s-wave meson-nucleon interaction in the $S=-1$ sector generates the $\Lambda(1405)$, and reproduces the $K^{-} p \rightarrow K^{-} p, \bar{K}^{0} n, \pi^{0} \Lambda, \pi^{0} \Sigma, \pi^{-} \Sigma^{+}$and $\pi^{+} \Sigma^{-}$cross-sections. The $\bar{K} N$ scattering amplitude in nuclear matter at finite tamperature is calculated within the coupled channel approach in Ref. [45]. This is evaluated incorporating the Pauli blocking, the dressing of the $\bar{K}$ (with s- and p-wave contributions) and $\pi$ meson as well as mean field binding of the nucleons and hyperons. The s- and p-wave contributions to the $\bar{K}$ self energy are due to the Weinberg-Tomozawa term and the coupling of $\bar{K}$ to the hyperon-hole excitations respectively, whereas the self-energy of the pion arises due to coupling of pion to particlehole (1p1h), $\Delta$-hole $(\Delta \mathrm{h})$ and two particle hole $(2 \mathrm{p} 2 \mathrm{~h})$ excitations. Due to Pauli blocking, the mass of $\mathrm{K}^{-}$is observed to increase upto a density of around $0.1 \rho$, which is observed to decrease with further increase in the density. When the $K^{-}$is dressed with s- and p-waves, there is a drop in the mass of the $K^{-}$, which is observed to be a smaller drop with density, when the pion self energy is also taken into account [11].

For the kaon-nucleon scattering, $K N$ is the only channel, and a self-consistent calculation 
is observed to lead to a positive mass shift of around $29 \mathrm{MeV}$ for $K$ meson in cold symmetric nuclear matter at nuclear matter saturation density, which is very close to the value of 25 MeV calculated using the $T \rho$ approximation. For $\bar{K}^{*}$ meson, the mass shift as obtained from the real part of the self energy $\left(\sim \operatorname{Re} \Pi\left(m_{\bar{K}^{*}}, \mathbf{q}=0\right) /\left(2 m_{\bar{K}^{*}}\right)\right)$ using a self consistent solution of the scattering amplitude within the coupled channel approach is observed to be around $-50 \mathrm{MeV}$ [46, 47], which is similar to the mass shift of the $\bar{K}$ meson [45]. For the $K^{*} N$ scattering, due to absence of any resonance near to the threshold, similar to $K N$ scattering, the low density $T \rho$ approximation is a good approximation for calculation of the self energy of the $K^{*}$ meson. The value of the mass shift for the $K^{*}$ meson of around $+50 \mathrm{MeV}$ at the nuclear matter saturation density obtained in the $T \rho$ approximation is modified to the value of around $+40 \mathrm{MeV}$ using the self-consistent calculation of the scattering amplitude [48]. The coupling of $K^{*} N$ to $K N$ has been considered in Ref. [49], which could arise from the decay process of $K^{*} \rightarrow K \pi$, followed by the pion decaying to a particle-hole pair, thus leading to the process $K^{*} N \rightarrow K N$. The $K^{*} N-K N$ coupling can modify the production of the $K$ and $K^{*}$ mesons resulting from nuclear collision experiments. However, no resonance formation arising due to the $K N-K^{*} N$ interaction is observed [49]. It might be mentioned here that the sign convention for the self energy in the dispersion relation of the $K$ and $\bar{K}$ meson used in the present work is opposite to the convention used in Refs. [10, 11, 45, 47, 50, 51]. The mass modifications of the hadrons with identical light $(u, d)$ quark (antiquark) constitutents, e.g., $K$ and $K^{*}$, are observed to be very similar within the QMC model [9]. This is due to the fact that these medium changes of the hadrons arise dominantly from the scalar potentials of the light quark (antiquark) constituents. In the present work, as has already been mentioned, we assume the mass shifts of $K^{*}\left(\bar{K}^{*}\right)$ mesons in the isospin asymmetric strange hadronic matter to be the same as the mass shifts of the $K(\bar{K})$ mesons as calculated using the chiral SU(3) model. This is motivated from the QMC model, where the mass shifts of the hadrons with the same nonstrange light quark and antiquark constituents are almost identical, arising dominantly from scalar potentials of these constituents [9]. The chiral SU(3) model used in the present work has been recently used to study the mass and decay width of the $\phi$ meson [52]. The values of the in-medium masses (in $\mathrm{MeV}$ ) of $K^{+}\left(K^{-}\right)$meson are observed to be around 525 (456) and 547 (311) for $\rho_{B}=\rho_{0}$ and $\rho_{B}=4 \rho_{0}$ respectively in symmetric nuclear matter $\left(\eta=0, f_{s}=0\right)$ in Ref. [17], which are used in the present work. These values may be compared with the values 
of around 524 (457) and 560 (312) $\mathrm{MeV}$ of Ref. [52] for the $K^{+}\left(K^{-}\right)$meson for the same densities in symmetric nuclear matter. One thus observes the values of the masses of the $K^{ \pm}$ in the present work to be very similar at $\rho_{B}=\rho_{0}$ with the values in Ref. [52], whereas, at the higher density of $\rho_{B}=4 \rho_{0}$, there is observd to be around $13 \mathrm{MeV}$ difference in the $K^{+}$mass (about 2\%) from the value in Ref. [52]. This is due to the frozen glueball approximation (the expectation value of the dilaton field $\chi$ kept fixed at its vacuum value, $\chi_{0}$ ), for the calculation of the scalar fields $(\sigma, \zeta$ and $\delta)$, using which the kaon and antikaon masses have been calculated in Ref. [17] and used in the present work. On the other hand, these scalar fields are calculated accounting for the medium dependence of the $\chi$ field [41, which are used for obtaining the in-medium masses of the $K$ and $\bar{K}$ mesons in Ref. [52], The mass of $K^{-}$at the higher density of $\rho_{B}=4 \rho_{0}$, however, is observed to be similar to the value in Ref. [52]. This is due to the reason that the $K^{-}$mass has negative contributions from the Weinberg-Tomozawa term, as well as, from the scalar fields $(\sigma, \zeta$ and $\delta$ ) (arising from the scalar exchange and (total) range terms), and the small modifications of these scalar fields including the medium modification of $\chi$ is observed to lead to only marginal modification to its mass in symmetric nuclear matter. In the presence of isospin asymmetry as well as strangeness fraction in the medium, the differences in the masses of kaons and antikaons in the present work from the values calculated in Ref. [52], are due to the fact that the masses used in the present work are obtained from the scalar fields $(\sigma, \zeta$ and $\delta)$, calculated assuming the frozen glueball approximation, whereas the medium modifications of the dilaton field, $\chi$ has been taken into account [41] for the calculations of the scalar fields [41] used for the study of the masses of the $K$ and $\bar{K}$ mesons in Ref. [52].

The modifications of the $\phi$ meson mass in hadronic medium calculated from the selfenergies of the $\phi$ meson due to kaon-antikaon loop [53], from QCD sum rule calculations [54, Quark meson coupling (QMC) model [8, 55] as well as other studies [56, 57] have been observed to be very small (at most 2-3\% at $\rho_{B}=\rho_{0}$ ). The mass modifications of the $\phi$ meson calculated from the $K-\bar{K}$ loop, using the in-medium masses of the kaons and antikaons obtained within the chiral effective model [52], is observed to be small (of the order of 2-3 $\mathrm{MeV})$ at $\rho_{B}=\rho_{0}$ in nuclear matter. In the present work, the in-medium spectral function as well as the production cross-section of the $\phi$ meson are studied without accounting for the mass modification of the $\phi$ meson.

The decay widths $K^{*}\left(\bar{K}^{*}\right) \rightarrow K(\bar{K}) \pi$ are computed using the field theoretic model of 
composite hadrons described in the previous section. These decay widths are obtained using equations 10 - 15), with $(\mathrm{A}, \mathrm{B}, \mathrm{C})=\left(K^{*}\left(\bar{K}^{*}\right), K(\bar{K}), \pi\right)$. The parameter, $\lambda_{2}$ in the expression for the decay width of $K^{*}\left(\bar{K}^{*}\right) \rightarrow K(\bar{K}) \pi$ is the fraction of the energy of the $K(\bar{K})$ meson carried by the constituent light $(\mathrm{u}, \mathrm{d})$ quark (antiquark). For the $K(\bar{K})$ at rest, the value of $\lambda_{2}$ is the fraction of the mass of the $K(\bar{K})$ meson carried by the constituent light quark (antiquark). In the present work, we take the constituent masses of light $(\mathrm{u}, \mathrm{d})$ and strange quarks to be $M_{u, d}=330 \mathrm{MeV}$ [31], $M_{s}=480 \mathrm{MeV}$ and the vacuum masses (in MeV) for charged (neutral) kaons (and antikaons) are taken as 493.68 (497.61) [25]. Assuming the binding energy of the meson shared by the quark (antquark) constituent to be inversely proportional to the quark (antiquark) mass [30], the value of the fraction of energy of the $K(\bar{K})$ carried by the light quark (antiquark), $\lambda_{2}$ is obtained as 0.71 [35]. The harmonic oscillator strengths of the $K$ and $\pi$ states are taken to be $R_{K}=(238.30 \mathrm{MeV})^{-1}, R_{\pi}=(210.81 \mathrm{MeV})^{-1}$ [30, 31] from the charge radii squared of pion and kaon to be $(0.63 \mathrm{fm})^{2}$ and $(0.56 \mathrm{fm})^{2}$ [25] respectively [35]. The value of $R_{\phi}$ is obtained from the observed decay width of $\phi \rightarrow e^{+} e^{-}$of $1.27 \mathrm{keV}$ [25] to be $(290.71 \mathrm{MeV})^{-1}$. The value of $R_{K^{*}}$ is calculated by assuming that $R_{K^{*}} / R_{K}=R_{K^{*}}^{b a g} / R_{K}^{b a g}$, where $R_{K^{*}}^{b a g}$ and $R_{K}^{b a g}$ are the bag radii of the $K^{*}$ and $K$ mesons of 0.74 fermis and 0.57 fermis calculated in the Quark meson coupling (QMC) model [9]. This yields the value of $R_{K^{*}}$ to be $184.84 \mathrm{MeV}$.

The values of $\gamma_{K^{*}}$ for the decays $K^{* \pm} \rightarrow(K(\bar{K}) \pi)^{ \pm}$and $K^{* 0}\left(\bar{K}^{*^{0}}\right) \rightarrow(K(\bar{K}) \pi)^{0}$ as fitted to their observed vacuum decay widths of 50.75 and $47.18 \mathrm{MeV}$ [25], are obtained as 2.44 and 2.36 respectively. These yield the values of the decay widths for the channels of the $K^{* \pm}$ meson to $K^{ \pm} \pi^{0}$ and $K^{0}\left(\bar{K}^{0}\right) \pi^{ \pm}$to be 16.89 and $33.86 \mathrm{MeV}$ and of decay widths of $K^{* 0}\left(\bar{K}^{*}\right)$ to $K^{0}\left(\bar{K}^{0}\right) \pi^{0}$ and $K^{ \pm} \pi^{\mp}$ to be 15.91 and $31.27 \mathrm{MeV}$ respectively.

In figure 2, the in-medium decay widths of the $K^{*} \rightarrow K \pi$ are plotted as functions of the baryon density for $\eta=0.3$ and $f_{s}=0,0.3,0.5$ and the results are compared with the isospin asymmetric case $(\eta=0)$. In panels (a) and (c), the decay widths of $K^{*+}$ meson are shown for the channels $K^{*+} \rightarrow K^{+} \pi^{0}$, and $K^{*+} \rightarrow K^{0} \pi^{+}$respectively, and the total decay width of $K^{*+} \rightarrow(K \pi)^{+}$, which is the sum of the two channels, is plotted in panel (e). Panels (b), (d) and (f) show the in-medium decay widths for the channels $K^{* 0} \rightarrow K^{0} \pi^{0}, K^{* 0} \rightarrow K^{+} \pi^{-}$, and, of $K^{* 0} \rightarrow(K \pi)^{0}$ (the sum of the two channels). The decay width of $K^{*+}$ to $K^{0} \pi^{+}$is observed to have much more pronounced effects from both the strangeness as well as isospin asymmetry of the hadronic medium, as compared to the decay width of $K^{*+}$ to $K^{+} \pi^{0}$. As 
might be observed from figure 1 , the isospin asymmetry leads to a drop (rise) of the mass of $K^{+}\left(K^{0}\right)$ meson (and of $K^{*+}\left(K^{* 0}\right)$ meson). This is observed as a substantial decrease in the decay width of $K^{*+}$ to $K^{0} \pi^{+}$as compared to $K^{*+}$ to $K^{+} \pi^{0}$ and an appreciable rise in the decay width of $K^{* 0}$ to $K^{+} \pi^{-}$as compared to $K^{* 0}$ to $K^{0} \pi^{0}$ in the isospin asymmetric matter as compared to the isospin symmetric $(\eta=0)$ case. The increase in the strangeness fraction in the hadronic medium is observed to lead to appreciable drop (increase) in the decay width of $K^{*+}\left(K^{* 0}\right)$ to $(K \pi)^{+}\left((K \pi)^{0}\right)$, as compared to the nuclear matter $\left(f_{s}=0\right)$ at high densities as can be observed from panels (e) and (f) in figure 2. These medium modifications of the masses of the $K^{*+}$ and $K^{* 0}$ mesons should modify the yield of these particles in the asymmetric heavy ion collision experiments.

In figure 3 , the decay widths of $\bar{K}^{*}$ to $(\bar{K} \pi)$ are plotted for the value of the isospin asymmetry parameter, $\eta=0.3$ and for typical values of the strangeness fraction. The results are compared to the isospin symmetric case. The decay widths for $K^{*-}$ to $K^{-} \pi^{0}, \bar{K}^{0} \pi^{-}$, and the sum of these two channels are plotted in panels (a), (c) and (e) respectively, whereas the decay widths for $\bar{K}^{*}{ }^{0}$ to $\bar{K}^{0} \pi^{0}, K^{-} \pi^{+}$, and the sum of these two channels are shown in panels (b), (d) and (f) respectively. The effects from the strangeness fraction on the decay widths of $\bar{K}^{*} \rightarrow \bar{K} \pi$ are observed to be rather moderate as compared to the effects on the $K^{*} \rightarrow K \pi$ decay widths shown in figure 2. There is observed to be a large increase in the decay width of the $K^{*-} \rightarrow \bar{K}^{0} \pi^{-}$as compared to that of $K^{*-} \rightarrow K^{-} \pi^{0}$, and a drop in the decay width of the $\bar{K}^{*^{0}} \rightarrow \bar{K}^{-} \pi^{+}$as compared to that of $\bar{K}^{*^{0}} \rightarrow \bar{K}^{0} \pi^{0}$ for the isospin asymmetric matter as compared to $\eta=0$ case. This is because of an increase (drop) of the mass of $\bar{K}^{0}\left(K^{-}\right)$(as well as for $\overline{K^{* 0}}\left(K^{*-}\right)$ with isospin asymmetry in the medium. In symmetric nuclear matter, the total decay width of $K^{*-} \rightarrow(\bar{K} \pi)^{-}$as well as of $\bar{K}^{* 0} \rightarrow(\bar{K} \pi)^{0}$ are observed to drop with increase in density. The values of these decay widths for $\rho_{B}=\rho_{0}\left(4 \rho_{0}\right)$ are obtained to be around 49.27 (37.67) and 43.28 (27.47) in symmetric nuclear matter. These results may be compared with the results of an appreciable increase in the decay width of $\bar{K}^{*}$ (around five times the vacuum value at the nuclear matter density), obtained within a coupled channel approach [46, 47]. The increase in the decay width within the coupled channel approach is due to the absorption channels $\bar{K}^{*} N \rightarrow \rho Y, \omega Y, \phi Y, Y=\Lambda, \Sigma$ [46, 47], in addition to the elastic channel $\bar{K}^{*} N \rightarrow \bar{K}^{*} N$ as well as accounting for the decay channel of $\bar{K}^{*} \rightarrow \bar{K} \pi$. The pion created from the decay $\bar{K}^{*} \rightarrow K \pi$, for example, can become a nucleon-hole pair in the medium, leading to the additional process of $\bar{K}^{*} N \rightarrow \bar{K} N$. Though the mass drop is 
observed to be moderate $(\sim 50 \mathrm{MeV})$ for $\bar{K}^{*}$ meson in the coupled channel approach, there is appreciable increase in its decay width due to coupling of $\bar{K}^{*} N$ to the hyperon-vector meson excitations, which can further increase due to processes $\bar{K}^{*} N \rightarrow \bar{K} N$. The in-medium decay width of $K^{*}\left(\bar{K}^{*}\right) \rightarrow K(\bar{K}) \pi$, which is the dominant decay channel of $K^{*}\left(\bar{K}^{*}\right)$ meson, is investigated in the present work from the mass modifications of the open strange vector and pseudoscalar mesons, but the mass modifications of the pion, which is observed to be small [45], has not been taken into account in the present work. The coupling of $\bar{K}^{*} N$ to the various coupled channels as mentioned above, which lead to appreciable increase in the decay width of the $\bar{K}^{*}$ meson [46, 47], are, however, beyond the scope of the mean field approach of the present work.

We next consider the medium modification of the decay width of $\phi \rightarrow K \bar{K}$. The values of $\gamma_{\phi}$ fitted from the observed decay widths of $\phi$ to the final states $K^{+} K^{-}$and $K^{0} \bar{K}^{0}$ in vacuum of 2.09 and $1.44 \mathrm{MeV}$ are obtained as 2.38 and 2.34 respectively. In figure 4 , the decay widths of $\phi$ meson to $K^{+} K^{-}$and $K^{0} \bar{K}^{0}$ are plotted in panels (a) and (b) for different values of the strangeness fraction, both for isospin symmetric $(\eta=0)$ as well as isospin asymmetric matter (with $\eta=0.3$ ). At the nuclear matter density, the decay width is observed to be $2.69,3.52$ and $3.90 \mathrm{MeV}$ for the channel (a) $\phi \rightarrow K^{+} K^{-}$, and, 3.55, 4.43 and 4.85 for the channel (b) $\phi \rightarrow K^{0} \bar{K}^{0}$ for the values of the strangeness fraction as $f_{s}=0,0.3$ and 0.5 respectively. The value of the total width of $6.24 \mathrm{MeV}$ at $\rho_{B}=\rho_{0}$ in symmetric nuclear matter in the present work may be compared with the value of around $25 \mathrm{MeV}$ in Ref. [53], a value of around 33-38 MeV (for the values of the momentum cut-off parameter chosen) within QMC model [55], and, the value of around $22 \mathrm{MeV}$ using a coupled channel approach [50], where the additional channels $\phi N \rightarrow K Y, Y=\Lambda, \Sigma$, as well as, $\phi N \rightarrow \Sigma^{*} K$, with $\Sigma^{*} \equiv \Sigma(1385)$ resonance, are incorporated through the p-wave self energy of $\bar{K}$ due to the coupling to the hyperon-hole $\left(Y N^{-1}\right)$ states, as well as, to the $\Sigma^{*} N^{-1}$ state. The in-medium decay widths of the $\phi$ mesons have also been studied experimentally from the study of the $\phi$ transperancy ratio of the photoprduction data at Spring8-LEPS [58] and CLAS [59] collaborations as well as the proton induced reactions in nuclei at COSY-ANKE [60]. In Ref. [60]. the $\phi$ mesons produced by collisions of protons with beam energy $2.83 \mathrm{GeV}$ on $\mathrm{C}, \mathrm{Cu}, \mathrm{Ag}, \mathrm{Au}$ targets detected via $\phi$ decaying to $K^{+} K^{-}$at ANKE detector at COSY were studied. The decay width of $\phi \rightarrow K^{+} K^{-}$extracted from the nuclear tranperancy ratio showed an appreciably large value (of the order of 33-50 MeV) of the in-medium decay wdith of $\phi \rightarrow K^{+} K^{-}$. In 
the present work of the composite model of hadrons, the values obtained for the decay width (in $\mathrm{MeV}$ ) of the $\phi \rightarrow K \bar{K}$ (sum of the channels with the neutral and charged $K \bar{K}$ ) final states) are around 6.24 and 25.11 in symmetric nuclear matter for $\rho_{B}=\rho_{0}$ and $\rho_{B}=3 \rho_{0}$ respectively, which are very similar to the the values calculated in Ref. [52] from the $K \bar{K}$ loop using the phenomenological interaction $\left(\sim \phi^{\mu}\left(\bar{K}\left(\partial_{\mu} K\right)-\left(\partial_{\mu} \bar{K}\right) K\right)\right.$ [53, 55]. The value of the decay width of $\phi \rightarrow K \bar{K}$ of around $6.24 \mathrm{MeV}$ at the nuclear matter saturation density in the present work as well as in Ref. [52] is thus observed to be much smaller than the value of around $22 \mathrm{MeV}$ in the coupled channel approach [50]. As has already been mentioned, the large value of the $\phi$ decay width arises due to the coupling $\phi N$ to the additional channels $\phi N \rightarrow K Y, Y=\Lambda, \Sigma$, as well as, $\phi N \rightarrow \Sigma^{*} K$, which is beyond the scope of the present work.

In the present work, as can be seen from figure 4 , the increase in the strangeness fraction of the hadronic medium leads to larger values of the decay widths of $\phi \rightarrow K \bar{K}$ (for both the channels (a) $\phi \rightarrow K^{+} K^{-}$as well as (b) $\phi \rightarrow K^{0} \bar{K}^{0}$ ). The effects of isospin asymmetry on the $\phi$ meson decay widths are observed to be rather moderate. However, the effect of the strangeness which is seen to lead to increase in the $\phi$ meson decay width and the rise in the decay width is observed to be quite pronounced at high densities. This should have observable consequences in the heavy ion collisions at the Compressed baryonic matter (CBM) experiments at future facility at GSI, e.g., the production of $K$ and $\bar{K}$ mesons to be more abundant as well as suppression of the $\phi$ meson. A drop in the production BreitWigner cross-sections of the $\phi$ meson (through the scattering of $K$ and $\bar{K}$ mesons) with the increase in the strangeness fraction of the medium, is observed, as might be seen from figure 9 .

In figures 5 and 6 , we show the effects of the strangeness and isospin asymmetry on the spectral functions of the $K^{*}\left(K^{*+}, K^{* 0}\right)$ and $\bar{K}^{*}\left(K^{*-}, \bar{K}^{*}\right)$ mesons for densities $\rho_{B}=\rho_{0}$ and $\rho_{B}=4 \rho_{0}$. In these figures, the vacuum spectral functions are shown as the dot-dashed curves. For nuclear matter $\left(f_{s}=0\right)$, there is observed to be a shift in the peak to a higher invariant mass for $K^{*}$ mesons from the vacuum value, whereas there is a downward shift as the strangeness fraction is increased. The isospin asymmetry leads to a higher (lower) value for the position of the peak for the $K^{* 0}\left(K^{*+}\right)$ spectral function. The observed behaviour is due to the assumption of the mass shifts of the $K^{*}$ mesons to be same as the mass shifts of the $K$ meson as calculated in the chiral $\mathrm{SU}(3)$ model. The spectral functions of the 

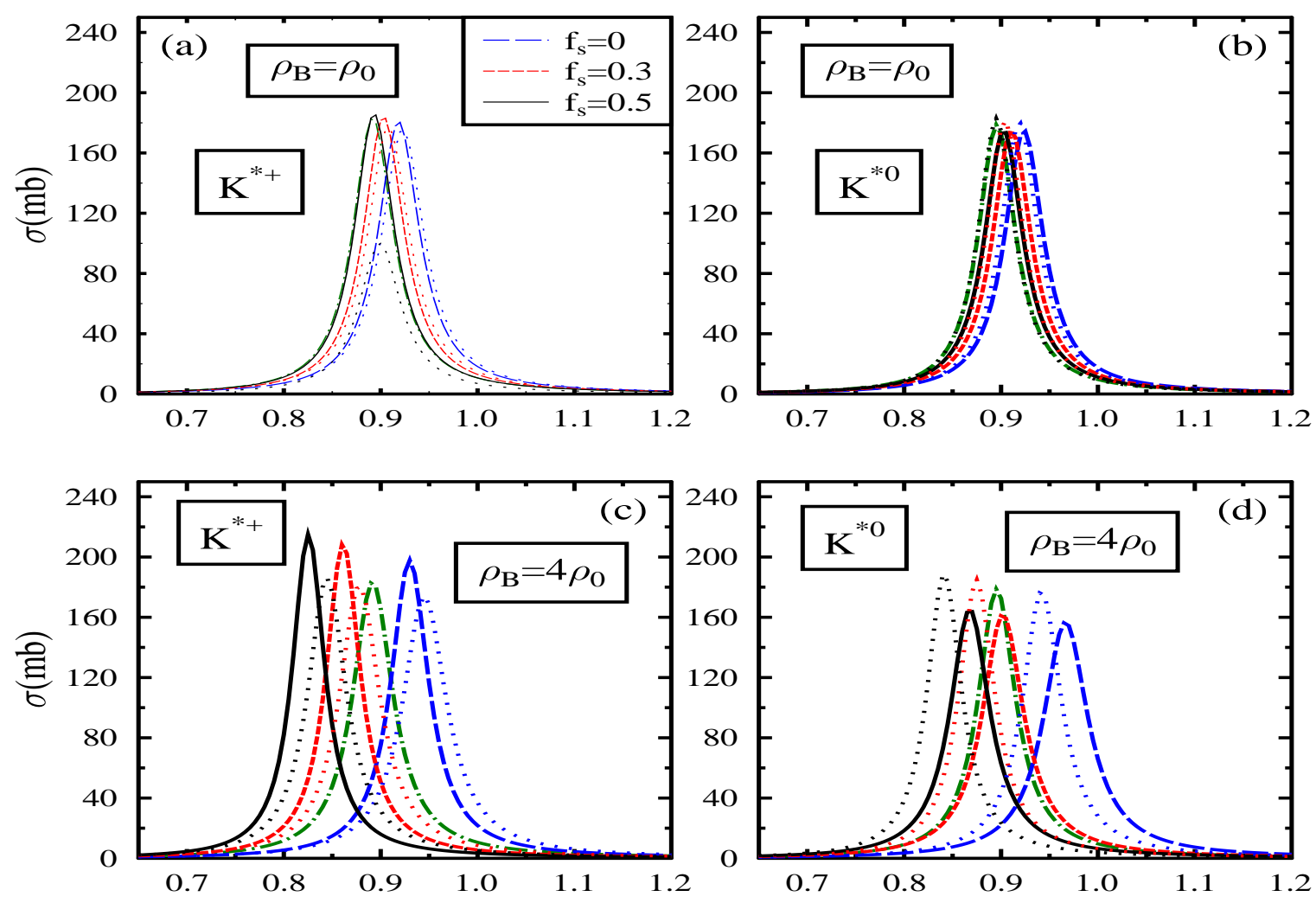

FIG. 7: Production Cross-section of the $K^{*}\left(K^{*+}, K^{* 0}\right)$ mesons for $f_{s}=0,0.3,0.5$ for isospin asymmetric matter with $\eta=0.3$. These are compared with results for symmetric matter $(\eta=0)$ shown as dotted lines. The production cross-section for the vacuum case is shown as the dotdashed line.

$\bar{K}^{*}\left(K^{*-}, \bar{K}^{*}\right)$ plotted in figure 6 show a much larger downward shift of the peak position for the higher value of density, $\rho_{B}=4 \rho_{0}$ as compared to $\rho_{B}=\rho_{0}$. The dependence on the isospin asymmetry as well as strangeness fraction are observed to be much more dominant 

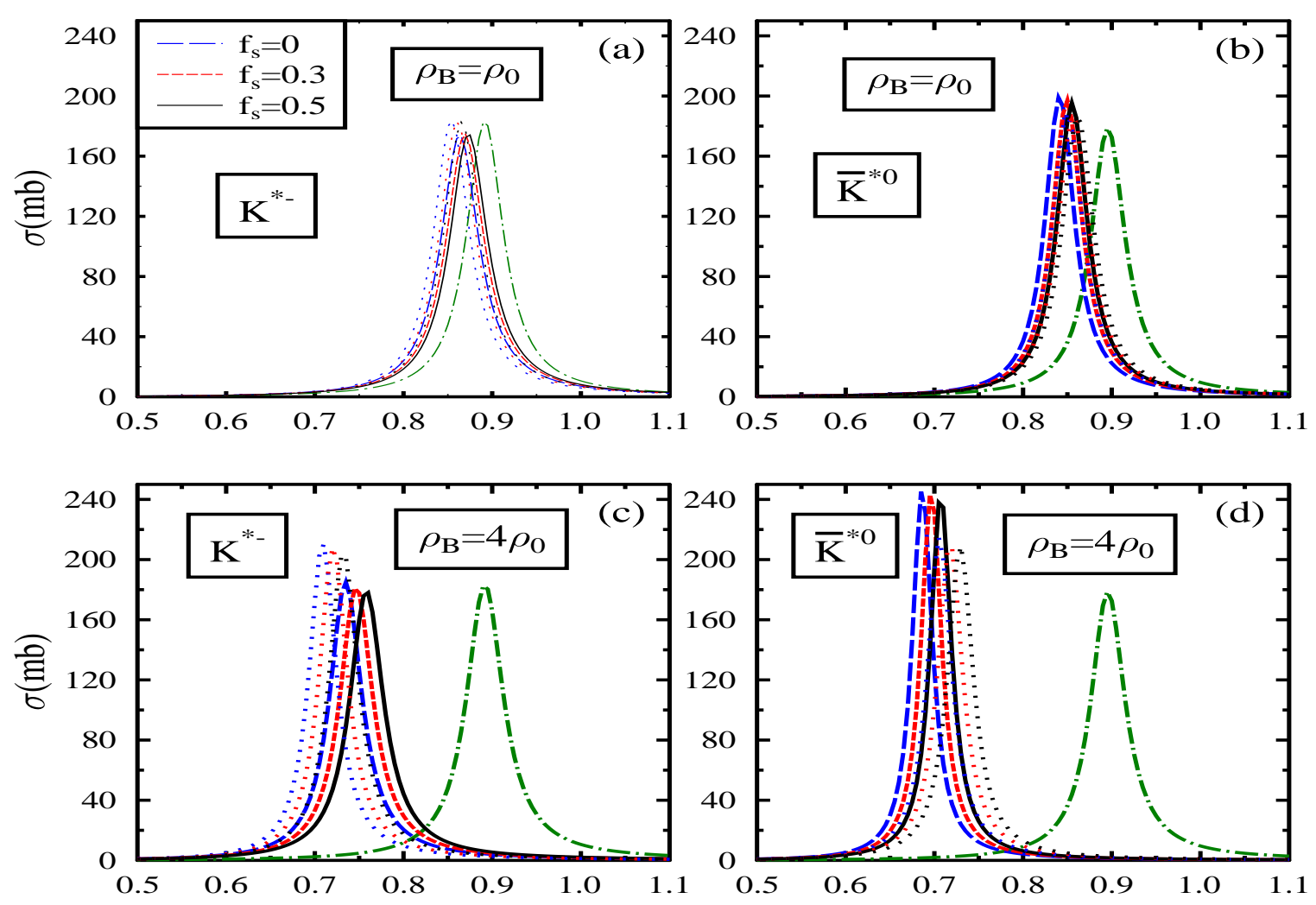

FIG. 8: Production Cross-section of the $\overline{K^{*}}\left(K^{*-}, \overline{K^{* 0}}\right)$ mesons for $f_{s}=0,0.3,0.5$ for isospin asymmetric matter with $\eta=0.3$ and compared with results for symmetric matter shown as dotted lines. The production cross-section for the vacuum case is shown as the dot-dashed line.

for the $K^{*+}$ and $K^{* 0}$ mesons as compared to the $K^{*-}$ and $\bar{K}^{*}{ }^{0}$ mesons. The spectral functions of the $K^{*}$ and $\bar{K}^{*}$ have been studied using the coupled channel approach in Refs. [13, 47]. For $\bar{K}^{*}$ meson, there is observed to be appreciable broadening of the quasi-particle peak due to the large increase in its decay width arising from the absorption channels 

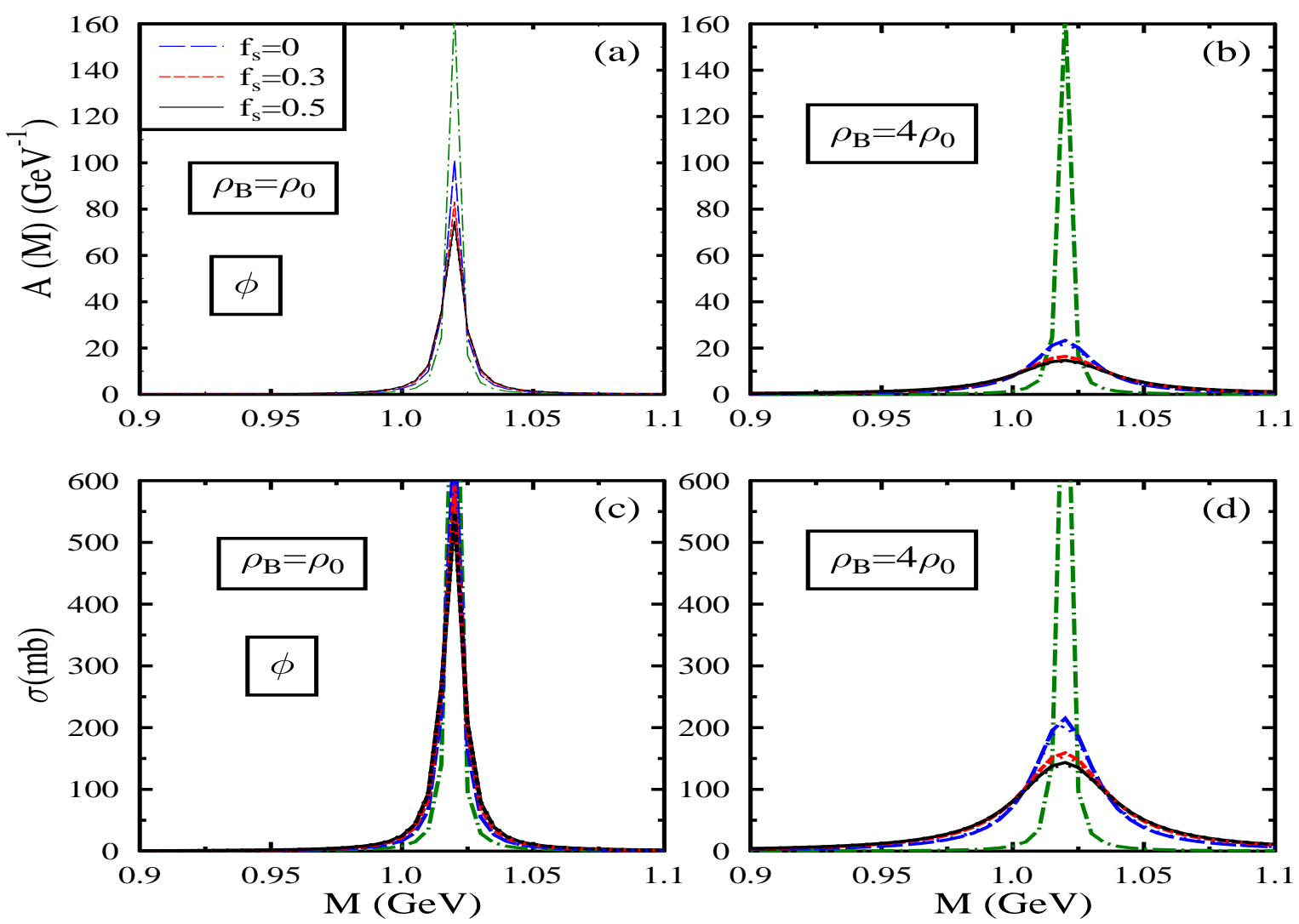

FIG. 9: Spectral functions and Production Cross-section of the $\phi$ mesons for $f_{s}=0,0.3,0.5$ for isospin asymmetric matter with $\eta=0.3$ and compared with results for symmetric matter $(\eta=0)$ shown as dotted lines. The spectral function as well as the production cross-section of $\phi$ meson for the vacuum case are shown as the dot-dashed lines.

$\bar{K}^{*} N \rightarrow V Y, V=\rho, \omega, \phi, Y=\Lambda, \Sigma$. There are two resonances generated dynamically, which can be identified with $\Lambda(1800)$ and $\Sigma(1750)$. The quasi-particle peak is moved to much lower energy due to the interference of the $\bar{K}^{*}$ with the resonance-hole excitations. In the absence of the absorption channels, the quasi-particle peak remains narrow for $K^{*}$ meson, which has 
contribution predominantly from the decay channel $K^{*} \rightarrow K \pi$.

Figures 7 and 8 , the production cross-sections for the $K^{*}$ and $\bar{K}^{*}$, as created from $K \pi$ and $\bar{K} \pi$ scatterings, are plotted. These are shown for densities $\rho_{0}$ and $4 \rho_{0}$. In the asymmetric hadronic matter with $\eta=0.3$, there is observed to be a modification in the maximum value of the production cross-section of $K^{*+}\left(K^{* 0}\right)$ meson from the vacuum value of $183 \mathrm{mb}$ to around 180 (174), 183 (175) and 185 (174) for $\rho_{B}=\rho_{0}$ and 198 (156), 208 (161) and 215 (164) for $\rho_{B}=4 \rho_{0}$, for $f_{s}=0,0.3$ and 0.5 respectively. There are observed to be marginal changes from the vacuum case for the maximum cross-section for $K^{*+}$ and $K^{* 0}$ mesons for symmetric hadronic matter $(\eta=0)$ shown as the dotted lines for both $\rho_{0}$ and $4 \rho_{0}$. For the $K^{*-}$ meson, the maximum value for the production cross-section (in milibarns) is observed to have small modifications from the vacuum value of 182 to around 174 and 185 in asymmetric nuclear matter (with $\eta=0.3$ ) for densities $\rho_{0}$ as well as $4 \rho_{0}$. However, the peak position for its production is at the lower invariant mass (in $\mathrm{GeV}$ ) of around 0.85 and 0.74 for these densities. In the absence of isospin asymmetry in the hadronic medium, the maximum values of the cross-sections are observed to be larger (smaller) than the isospin asymmetric case for $K^{*-}\left(\bar{K}^{*}\right)$ mesons respectively. The maximum cross-section for $\bar{K}^{*}$ is observed to be around $199 \mathrm{mb}$ at nuclear matter saturation density and around $245 \mathrm{mb}$ at $\rho_{B}=4 \rho_{0}$ in asymmetric nuclear matter. The modifications of $\sigma_{\max }$ is observed to be marginal with finite strangeness fraction in the medium. The production cross-sections of the $K^{*+}$ as compared to $K^{* 0}$ meson from $K \pi$ scattering as well as of $\bar{K}^{*}{ }^{0}$ as compared to $K^{*-}$ from $\bar{K} \pi$ scattering in the isospin asymmetric hadronic medium at high densities, should show in the $K^{*+} / K^{* 0}$ and $\bar{K}^{*^{0}} / K^{*-}$ ratios in the asymmetric heavy ion collision in the compressed baryonic experiment at the future facility at GSI.

Figure 9 shows the spectral function as well as the production cross-section of $\phi$ meson due to $K \bar{K}$ scattering. One observes a broadening of the peak for the higher density of $4 \rho_{0}$ as compared to the nuclear matter density. The maximum value of the production crosssection (in milibarns) is observed to have a substantial reduction to around 205 (215), 154 (159) and $140(144)$ for $f_{s}=0,0.3$ and 0.5 respectively at the higher baryon density of $4 \rho_{0}$ for hadronic matter with $\eta=0$ (0.3). This should be observed as suppression of $\phi$ meson in the hadronic medium at high densities. 


\section{SUMMARY}

We have investigated the in-medium spectral functions as well as the production crosssections of strange vector mesons $\left(K^{*}, \bar{K}^{*}, \phi\right)$ in isospin asymmetric nuclear (hyperonic) matter. The in-medium decay widths of these mesons, for the decay processes $K^{*} \rightarrow K \pi$, $\bar{K}^{*} \rightarrow \bar{K} \pi$, and $\phi \rightarrow K \bar{K}$ are computed from the medium modifications of the masses of the open strange mesons, using a field theoretic model of composite hadrons with quark (antiquark) constituents. These are calculated from the matrix element of the quark-antiquark pair creation term of the free Dirac Hamiltonian between the inital and final states. The masses of the kaons and antikaons are calculated using a chiral SU(3) model and the mass shift of the vector $K^{*}\left(\bar{K}^{*}\right)$ meson is assumed to be the same as the mass shift of the $K(\bar{K})$ meson in the hadronic medium. There is observed to be dominant contributions to the kaon and antikaon masses from the isospin asymmetry as well as strangeness at high densities, which is observed to lead to large mass difference of the $K^{+}$and $K^{0}$ mesons. This should show in the experimental observable, e.g, the $K^{+} / K^{0}$ ratio in asymmetric heavy ion collision expertiments. There are observed to be significant modifications of the decay widths of the $K^{*}\left(K^{*+}, K^{* 0}\right)$, and $\bar{K}^{*}\left(K^{*-}, \bar{K}^{* 0}\right)$, from the decay processes $K^{*} \rightarrow K \pi$ and $\bar{K}^{*} \rightarrow \bar{K} \pi$ in the isospin asymmetric strange hadronic matter and the decay width of $\phi \rightarrow K \bar{K}$ is observed to be appreciably larger with increase in strangeness in the medium.

The effects of the strangeness and isospin asymmetry on the spectral functions as well as the production cross-sections of the $K^{*}, \bar{K}^{*}$ and $\phi$ mesons arising from the $K \pi, \bar{K} \pi$ and $K \bar{K}$ scatterings respectively, should show in the yields of these particles. The substantial increase in the production cross-section of $K^{*+}$ as compared to $K^{* 0}$ meson, of $\bar{K}^{*}$ as compared to $K^{*-}$, as well as of $\phi$ in the isospin asymmetric hadronic medium at high densities, should show in the yields of these particles in asymmetric heavy ion collisions at the compressed baryonic matter (CBM) experiments at the future facility at GSI.

One of the authors (AM) acknowledeges financial support from Department of Science and Technology (DST), Government of India (project no. CRG/2018/002226).

[1] C. Hartnack, H. Oeschler, Y. Leifels, E. L. Bratkovskaya, J. Aichelin, Phys. Rep. 510, 119 (2012). 
[2] E. Friedman and A. Gal, Phys. Rep. 452, 89 (2007).

[3] L. Tolos and L. Fabbietti, Prog. Nucl. Part. Phys 112, 103770 (2020).

[4] D. B. Kaplan and A. E. Nelson, Phys. Lett. B 175, 57 (1986); A. E. Nelson and D. B. Kaplan, ibid, 192, 193 (1987).

[5] N. K. Glendenning and J. Schaffner-Bielich, Phys. Rev. C 60, 025803 (1999).

[6] S. Banik, R. Nandi, D. Bandyopadhyay, Phys. Rev. C 86, 045803 (2012).

[7] B.D. Serot, Rep. Prog. Phys. 55, 1855 (1992).

[8] K. Tsushima, K. Saito, A.W. Thomas, S.V. Wright, Phys. Lett. B 429, 239 (1998); ibid, Phys. Lett. E 436, 453 (1998); K. Tsushima, A. Sibirtsev, A.W. Thomas, Phys. Rev. C 62064904 (2000); ibid, J. Phys. G 27, 34 (2001).

[9] G. Krein, A. W. Thomas, K. Tsushima, Prog. Part. Nucl. Phys.100, 161 (2018).

[10] . E. Oset and A. Ramos, Nucl. Phys. A 635, 99 (1998).

[11] A. Ramos, E. Oset, Nucl. Phys. A 671,481 (2000).

[12] A. Ilner, D. Cabrera, C. Markert and E. Bratkovskaya, Phys. Rev. C 95, 014903 (2017).

[13] A. Ilner, J. Blair, D. Cabrera, C. Markert and E. Bratkovskaya, Phys. Rev. C 99, 024914 (2019).

[14] A. Mishra, E. L. Bratkovskaya, J. Schaffner-Bielich, S. Schramm and H. Stöcker, Phys. Rev. C 70, 044904 (2004).

[15] A. Mishra and S. Schramm, Phys. Rev. C 74, 064904 (2006).

[16] A. Mishra, S. Schramm and W. Greiner, Phys. Rev. C 78, 024901 (2008).

[17] A. Mishra, A. Kumar, S. Sanyal, S. Schramm, Eur. Phys. J A 41, 205 (2009).

[18] P. Papazoglou, D. Zschiesche, S. Schramm, J. Schaffner-Bielich, H. Stöcker, and W. Greiner, Phys. Rev. C 59, 411 (1999).

[19] A. Mishra, K. Balazs, D. Zschiesche, S. Schramm, H. Stöcker, and W. Greiner, Phys. Rev. C 69, 024903 (2004).

[20] S.Weinberg, Phys. Rev. 1661568 (1968).

[21] S. Coleman, J. Wess, B. Zumino, Phys. Rev. 1772239 (1969); C.G. Callan, S. Coleman, J. Wess, B. Zumino, Phys. Rev. 1772247 (1969).

[22] W. A. Bardeen and B. W. Lee, Phys. Rev. 1772389 (1969).

[23] J. Schechter, Phys. Rev. D 21, 3393 (1980).

[24] J.Ellis, Nucl. Phys. B 22, 478 (1970); B. A. Campbell, J. Ellis and K. A. Olive, Nucl. Phys. 
B 345,57 (1990).

[25] P. A. Zyla et al (Particle Data Group), Prog. Theor. Exp. Phys. 2020, 083C01 (2020).

[26] G. E. Brown, C.-H. Lee, M. Rho, and V. Thorsson, Nucl. Phys. A 567, 937 (1994).

[27] J. Schaffner-Bielich, I. N. Mishustin, J. Bondorf, Nucl. Phys. A 625, 325 (1997).

[28] T. Barnes and E. S. Swanson, Phys. Rev. C 49, 1166 (1994).

[29] S. P. Misra, Phys. Rev. D 18, 1661 (1978).

[30] S. P. Misra, Phys. Rev. D 18, 1673 (1978).

[31] Amruta Mishra, S. P. Misra and W. Greiner, Int. J. Mod. Phys. E 24, 1550053 (2015).

[32] Amruta Mishra and S. P. Misra, Phy. Rev. C 95, 065206 (2017).

[33] Amruta Mishra and S. P. Misra, Phys. Rev. C 102, 045204 (2020).

[34] Amruta Mishra and S. P. Misra, arXiv:2005.00354 (hep-ph).

[35] Amruta Mishra and S. P. Misra, arXiv:2006.03478 (nucl-th), to be published in Int. Jour. Mod. Phys. E.

[36] E.S.Ackleh, T. Barnes and E. S. Swanson, Phys. Rev. D 54, 6811 (1996).

[37] T. Barnes, F. E. Close, P. R. Page and E. S. Swanson, Phys. Rev. D 55, 4157 (1997).

[38] A. Le Yaouanc, L. Oliver, O. Pene and J.-C. Raynal, Phys. Rev. D 8 2223, (1973); ibid, Phys. Rev. D 9, 1415 (1974); ibid, Phys. Rev. D 11, 1272 (1975).

[39] A. Le Yaouanc, L. Oliver, O. Pene and J.-C. Raynal, Phys. Lett. B 71397 (1977).

[40] B. Friman, S. H. Lee and T. Song, Phys. Lett, B 548, 153 (2002).

[41] Arvind Kumar and Amruta Mishra, Eur. Phys. Jour. A 47, 164 (2011).

[42] A. Ilner, D. Cabrera, P. Srisawad and E. Bratkovskaya, Nucl. Phys. A 927, 249 (2014).

[43] K. Haglin, Nucl. Phys. A 584, 719 (1995).

[44] G. Q. Li, C. M . Ko and G. E. Brown, Nucl. Phys. A 611, 539 (1996).

[45] L. Tolos, D. Cabrera and A. Ramos, Phys. Rev. C 78, 045205 (2008).

[46] L. Tolos, R. Molina, E. Oset and A. Ramos, Phys. Rev. C 82, 045210 (2010).

[47] L. Tolos, D. Cabrera, R. Molina, E. Oset and A. Ramos, Prog. Theor. Phys. Suppl. 186, 384 (2010), contribution to New Frontiers in QCD 2010: Exotic Hadron Systems and Dense Matter.

[48] D. Cabrera, L. M. Abreu, E. Bratkovskaya, A. Ilner, F. J. Llanes-Estrada, A. Ramos, L. Tolos, J. M. Torres-Rincon, J. Phys. Conf. Ser. 503, 012017 (2014), Contribution to FAIRNESS 2013.

[49] K. P. Khemchandani, A. Martinez Torres, F. S. Navarra, M. Nielsen, L. Tolos, Phys. Rev. D 
91, 094008 (2015).

[50] E. Oset and A. Ramos, Nucl. Phys. A 679, 616 (2001).

[51] E. Oset and A. Ramos, Eur. Phys. J. A 44, 445 (2010).

[52] R. Kumar and A. Kumar, Phys. Rev. C 102, 045206 (2020).

[53] C. M. Ko, P. Levai, X.J. Qiu, C.T. Li, Phys. Rev. C 45, 1400 (1992).

[54] T. Hatsuda, S.H. Lee, Phys. Rev. C 46, R34 (1992). T. Hatsuda, H. Shiomi, H. Kuwabara, Progr. Theoret. Phys. 95, 1009 (1996).

[55] J.J. Cobos-Martinez, K. Tsushima, G. Krein and A. W. Thomas, Phys. Lett. B 771, 113 (2017).

[56] F. Klingl, T. Waas, W. Weise, Phys. Lett. B 431, 25 (1998).

[57] D. Cabrera, M.J. Vicente Vacas, Phys. Rev. C 67, 045203 (2003).

[58] T. Ishikawa et al, Phys. Lett. B 608, 215 (2005).

[59] M. H. Wood et al, Phys. Rev. Lett. 105, 112303 (2010).

[60] M. Hartmann et al, Phys. Rev. C 85, 035206 (2012). 